\title{
FY20 BISON BWR Fuel Modeling Capability: Material Models
}

\section{September 2020}

Technical Report

Aysenur Toptan ${ }^{1}$ and Kyle Gamble ${ }^{1}$

${ }^{1}$ Idaho National Laboratory

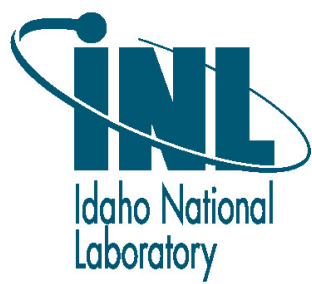




\section{DISCLAIMER}

This information was prepared as an account of work sponsored by an agency of the U.S. Government. Neither the U.S. Government nor any agency thereof, nor any of their employees, makes any warranty, expressed or implied, or assumes any legal liability or responsibility for the accuracy, completeness, or usefulness, of any information, apparatus, product, or process disclosed, or represents that its use would not infringe privately owned rights. References herein to any specific commercial product, process, or service by trade name, trade mark, manufacturer, or otherwise, does not necessarily constitute or imply its endorsement, recommendation, or favoring by the U.S. Government or any agency thereof. The views and opinions of authors expressed herein do not necessarily state or reflect those of the U.S. Government or any agency thereof. 


\title{
FY20 BISON BWR Fuel Modeling Capability: Material Models
}

\author{
Technical Report \\ Aysenur Toptan ${ }^{1}$ and Kyle Gamble ${ }^{1}$ \\ ${ }^{1}$ Idaho National Laboratory
}

30 September 2020

Idaho National Laboratory

Computational Mechanics and Materials Department

Idaho Falls, Idaho 83415

http://www.inl.gov

Prepared for the

U.S. Department of Energy

Office of Nuclear Energy

Under U.S. Department of Energy-Idaho Operations Office

Contract DE-AC07-05ID14517 
Page intentionally left blank 


\section{Abstract}

Until this year, the BISON fuel performance code has been developed primarily for pressurized water reactor (PWR), accident tolerant fuel (ATF), and advanced reactor (e.g., tri-structural isotropic (TRISO), metallic) analyses. The main focus of this work is to develop and document required material models that would aid predicting cladding failure with a specific hydride distribution to be investigated for boiling water reactor (BWR) applications in the next fiscal year. In terms of materials, the primary differences between a BWR and PWR is the use of gadolinia doping of the $\mathrm{UO}_{2}$ fuel, the use of Zircaloy-2 for the cladding, and the addition of a liner on the inner surface of the cladding for improved pellet-clad mechanical interaction (PCMI)

performance. The liner is typically made of pure zirconium or a lower tin content zirconium alloy (e.g. Zr$0.3 \% \mathrm{Sn}$ ). This work details the development and documentation of material and behavioral models for BWR analysis such as the addition of material and behavior models for gadolinia doped $\mathrm{UO}_{2}$, pure zirconium, Zr-0.3\%Sn, and Zircaloy-2 as well as the ease-of-use additions to allow internal meshing capabilities to include liners. Another important aspect affecting cladding performance is hydrogen diffusion and hydride precipitation in the cladding. A model was added previously for non-lined, homogeneous claddings. In this work, instructions are provided on how to apply the model the BWR liner cladding. Representative experimental values are provided for the hydrogen diffusion parameters and precipitation-dissolution kinetics for typical liner materials of interest. 
Page intentionally left blank 


\section{Acknowledgments}

This research is supported by and performed in conjunction with the Consortium for Advanced Simulation of Light Water Reactors, an Energy Innovation Hub for Modeling and Simulation of Nuclear Reactors under U.S. Department of Energy Contract No. DE-AC07-05ID14517.

This research made use of the resources of the High Performance Computing Center at Idaho National Laboratory, which is supported by the Office of Nuclear Energy of the U.S. Department of Energy and the Nuclear Science User Facilities under Contract No. DE-AC07-05ID14517. 
Page intentionally left blank 


\section{Contents}

Abstract

List of Figures $\quad$ x

List of Tables

List of Symbols

List of Listings $\quad$ xii

Acronyms

1 Introduction 2

2 Materials/Behavioral Models 4

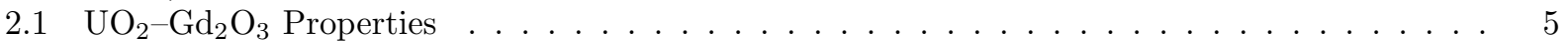

2.2 Pure Zirconium Properties . . . . . . . . . . . . . . . . . . . . . . . . . . . . 10

2.3 Zirconium Tin Properties . . . . . . . . . . . . . . . . . . . . . . . 11

2.4 Zircaloy-2 Properties . . . . . . . . . . . . . . . . . . . . . . . . . . . . . 12

2.5 Hydrogen Migration/Redistribution Model of BISON . . . . . . . . . . . . . . . . . . . 15

3 Liner Developments $\quad \mathbf{2 0}$

3.1 Internal Meshing Capabilities . . . . . . . . . . . . . . . . . . . . . . . . 20

3.2 BISON Mesh Script . . . . . . . . . . . . . . . . . . . . . . . 22

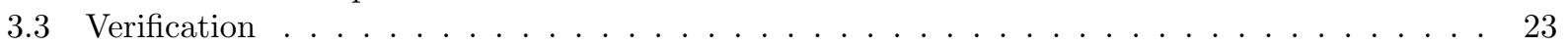

4 Validation $\mathbf{2 6}$

4.1 Experiment: IFA-681 Rods 1, 2, and $3 \ldots \ldots$. . . . . . . . . . . . . . 26

4.2 BISON Model Settings . . . . . . . . . . . . . . . . . . . . . . . . 27

4.3 Results and Discussion . . . . . . . . . . . . . . . . . . . . . . 31

5 Concluding Remarks 32

6 Publications 35

$\begin{array}{ll}\text { Bibliography } & 37\end{array}$

Appendices $\quad 41$ 
A Gap Conductance: Thermal Aspects 


\section{List of Figures}

2.1 Comparison of the thermal conductivity model at $T=T_{i r r}$ using (a) the Ronchi model, (b) the Staicu model, and (c) the Staicu model with an arbitrary gadolinia concentration of 10wt.\%. The thermal conductivity increases in the transition region where the high burnup structure (HBS) commences to form. [13] attributes the increase in the thermal conductivity in the HBS formation to the decrease in the fission gas concentration dissolved in the matrix. The degradation of the thermal conductivity resumes as a result of the accumulation of fission products for further increase of the burnup. . . . . . . . . . . . . 7

2.2 Specific heat capacity of $\mathrm{UO}_{2}$ at arbitrarily chosen gadolinia concentrations (0 to $\left.20 \mathrm{wt} . \%\right)$. 9

2.3 Schematic illustration of hydride migration and redistribution within a Zircaloy cladding . . . 16

3.1 A Layered1D mesh containing fuel and lined cladding. . . . . . . . . . . . . . . . . . 21

3.2 A smeared pellet mesh containing a lined cladding. . . . . . . . . . . . . . . . . 22

3.3 Discrete pellet mesh containing dishes and chamfers with a lined cladding. . . . . . . . . . 23

3.4 Temperature distribution for the verification problem. Exact and finite element (FE) solutions are obtained using one-dimensional elements. . . . . . . . . . . . . . . . . 25

4.1 The average linear heat rate histories for the Halden IFA-681 irradiation. . . . . . . . . . 28

4.2 A front-view of the IFA-681.1 rod 1 (left) and a close-up view of the IFA-681.1 rod 1, a cut from the top of the fuel $\operatorname{rod}($ right $) \ldots \ldots \ldots \ldots \ldots \ldots \ldots \ldots$

4.3 Halden IFA-681.1 fuel temperature histories at thermocouple (TC) locations. . . . . . . . . 31

5.1 Gantt diagram for $2020-2021$ fiscal years . . . . . . . . . . . . . . . . . . . . . . 34

6.1 Comparisons of model predictive envelopes for the stoichiometric $\mathrm{UO}_{2}$ thermal conductivity in (a) the burnup range varied from 0 to $60 \mathrm{MWd} / \mathrm{kgU}$ and (b) the gadolinia concentration varied from 0 to $10 \mathrm{wt} \%$. . . . . . . . . 


\section{List of Tables}

2.1 Material models available for BWR analyses. . . . . . . . . . . . . . . . . . . . . . . 4

2.2 A list of the conventional Gd-doped $\mathrm{UO}_{2}$ thermal conductivity models in BISON. . . . . . . 8

2.3 The empirical constants used in Eq. 2.17 from [17]. . . . . . . . . . . . . . . . . . . . . . 9

2.4 Coefficients used in the polynomial fits for the material constants used in the $\mathrm{Zr}-0.3 \% \mathrm{Sn}$ creep correlation. . . . . . . . . . . . . . . . . . . . . . . 11

2.5 Specific heat capacities for Zircaloy. . . . . . . . . . . . . . . . . . . . . . . . . 12

2.6 Parameters for high temperature creep of Zircaloy-2. . . . . . . . . . . . . . . . . . . . . . . 15

2.7 BISON cladding hydrides action parameters . . . . . . . . . . . . . . . . . . . 18

2.8 Representative experimental values taken from $[25$, Table $1-3]$. . . . . . . . . . . . . . . 19

4.1 Fuel rod specifications for IFA-681 $[52,53,54] . \ldots$. . . . . . . . . . . . . . . . 27 


\section{Listings}

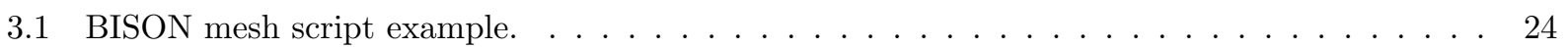




\title{
List of Symbols
}

\author{
Greek Letters \\ $\alpha \quad$ Thermal diffusivity $\left(=k / \rho c_{P}\right)$ \\ $\nu \quad$ Poisson's ratio \\ $\theta \quad$ Volume fraction of hydrogen in the $\alpha$-phase \\ Roman Letters \\ A Area \\ $B u \quad$ Burnup \\ C Concentration \\ $c_{P} \quad$ Specific heat capacity \\ $D_{0} \quad$ Diffusion frequency factor \\ E Young's modulus \\ $E_{D} \quad$ Activation energy \\ $E_{G} \quad$ Activation energy for reaction-driven growth \\ $f_{\text {clamp }}$ Factor limiting the maximum hydride content in a node \\ $G \quad$ Shear modulus \\ Gd Gadolinia content \\ $k \quad$ Thermal conductivity \\ $K_{D 0} \quad$ Coefficient for dissolution kinetics \\ $K_{\text {difo }} \quad$ Coefficient for diffusion-driven growth kinetics \\ $K_{N h 0} \quad$ Coefficient for nucleation kinetics \\ $K_{t h 0} \quad$ Coefficient for reaction-driven growth kinetics \\ $Q^{\star} \quad$ Heat of transport \\ $Q_{D} \quad$ Activation energy for the solubility \\ $Q_{P} \quad$ Activation energy for the super-solubility \\ $R \quad$ Universal gas constant $(=8.3143)$ \\ $T \quad$ Temperature \\ $T S S_{D 0}$ Coefficient/frequency factor for the solubility \\ $T S S_{P 0}$ Coefficient/frequency factor for the super-solubility \\ Subscripts \\ $\infty \quad$ Ambient condition \\ prec Precipitated hydrogen as hydrides \\ ss Dissolved hydrogen in the solid solution \\ $95 \quad$ At $95 \%$ theoretical density \\ el Electronic \\ eol End of life \\ ph Phonon-phonon interaction \\ self Self-irradiation
}

$\mathrm{m}^{2} / \mathrm{s}$ 


\title{
Acronyms
}

\author{
ALHR average linear heat rate \\ ATF accident tolerant fuel \\ BWR boiling water reactor \\ CASL Consortium for Advanced Simulation of Light Water Reactors \\ DOE U.S. Department of Energy \\ EOL end of life \\ FE finite element \\ FGR fission gas release \\ HBS high burnup structure \\ HBWR Halden Boiling Water Reactor \\ HNGD Hydride Nucleation - Growth - Dissolution Model \\ IFE Institutt for Energiteknikk \\ INL Idaho National Laboratory \\ LHR linear heat rate \\ LWR light water reactor \\ MOOSE Multiphysics Object-Oriented Simulation Environment \\ PCMI pellet-clad mechanical interaction \\ PWR pressurized water reactor \\ RIA reactivity insertion accident \\ SQA software quality assurance \\ TC thermocouple \\ TD theoretical density \\ TRISO tri-structural isotropic \\ VERA Virtual Environment for Reactor Applications
}


Page intentionally left blank

1 of 42 


\section{Introduction}

In 2010, the U.S. Department of Energy (DOE) created its first Energy Innovation Hub, which is focused on developing high-fidelity and high-resolution modeling and simulation tools for the modeling of light water reactors (LWRs). This hub, the Consortium for Advanced Simulation of Light Water Reactors (CASL) ${ }^{1}$, has developed an LWR simulation tool called the Virtual Environment for Reactor Applications (VERA). BISON is the high-fidelity and high-resolution fuel performance tool used in VERA.

BISON $[1,2,3]$ is a fuel performance code that models the thermo-mechanical behavior of nuclear fuel using high performance modeling and simulation. BISON is established on the Multiphysics Object-Oriented Simulation Environment (MOOSE) framework ${ }^{2}[4,5]$ of Idaho National Laboratory (INL). BISON solves the fully-coupled equations of energy conservation, mechanics, and species conservation to account for a large range of different fuel behavior. It is capable of modeling traditional LWR fuel rods, fuel plates, and tristructural isotropic (TRISO) fuel particles [6, 7]. It can employ three-dimensional Cartesian, two-dimensional axisymmetric, two-dimensional generalized plane strain, layered two-dimensional, layered one-dimensional, one-dimensional radial spherical geometries. It includes empirical models for a large variety of fuel thermal and mechanical physics.

BWR fuel differs from PWR fuel in terms several aspects, considering (a) extensive use of gadolinia up to 10 wt.\%, which significantly impacts the fuel thermal conductivity, consequentially on the fuel temperature distribution; and (b) the use of different alloying materials such as the fuel liners (e.g., pure zirconium or zirconium tin), to increase the margin to PCMI failure, enabling more flexible operation. Such liners tend to exhibit much higher hydrogen contents than the rest of the cladding (in the hottest side of the cladding wall). The concentration of hydrogen and consequent localized precipitation of hydrides can cause the failure of the cladding at small strains.

This study focuses on expanding BISON's BWR fuel modeling capability by developing needed material models that would aid in predicting cladding failure with a specific hydride distribution, taking into account the hydride geometry and hydrogen distribution within the liner cladding. As a part of the work scope, Fiscal Year 2020 (FY2020) focused on the addition of materials modeling to allow the use of different alloying materials and gadolinia up to 10 wt.\%. The tasks to be completed in FY2020 are: (1) to complete an initial assessment of material model needs for BWR fuel, which is completed prior to this milestone report; and

\footnotetext{
${ }^{1}$ www.casl.gov

${ }^{2}$ a high-performance, open source, C++ FE framework: www.github.com/idaholab/moose
} 
(2) to develop and document material models for BWR fuel in BISON including verification and validation, which is the scope of this milestone report.

This report is organized as follows: Chapter 2 describes BISON's modeling options of the $G d$-bearing $\mathrm{UO}_{2}$ thermal properties as well as the liner materials, such as pure zirconium, zirconium tin, and Zircaloy2 thermal and/or mechanical properties in addition to the behavioral model for the hydrogen migration and redistribution within a Zircaloy cladding. Chapter 3 outlines BISON's internal meshing capabilities for the liner layer being added to the inner face of cladding. Chapter 4 covers the validation activities in which BISON's predictions are compared to the measurements from Halden IFA-681.1 experiment. Chapter 5 concludes the document with a discussion of the results and future work. Lastly, the publication documenting BISON's new thermal conductivity capability for gadolinia-bearing uranium dioxide fuel prior to the addition of the latest models described within this report are highlighted in Chapter 6. 


\section{Materials/Behavioral Models}

In this chapter, the material property models of BISON for BWR fuel rod analysis are documented, which are tabulated in Table 2.1. The materials of interest are uranium dioxide fuel with gadolinia, $\mathrm{Gd}_{2} \mathrm{O}_{3}$, addition (see Section 2.1) and liner cladding material models, such as pure zirconium (see Section 2.2), zirconium tin (see Section 2.3), and/or Zircaloy-2 properties (see Section 2.4). Lastly, the new features are being documented, integrated into the BISON test suite, and run periodically to ensure that code capabilities are not lost. It should be noted that models for low tin content alloys, as with pure zirconium for nuclear applications, are scarce in the open literature. In absence of a specific model for liners, Zircaloy-2 properties can be used. All of the Zircaloy-2 models presented in this chapter are existing models of BISON and are provided for the purposes of completeness. Additional properties for $\mathrm{UO}_{2}-\mathrm{Gd}_{2} \mathrm{O}_{3}$ fuels are not presented here, owing to the absence properties in the literature; the assumption is to use the same material models as those used for $\mathrm{UO}_{2}$ without the gadolinia addition. Lastly, an important physics (a so-called behavioral model) to consider for BWR fuel rod behavior is the hydrogen migration and redistribution model, which is briefly outlined in Section 2.5 .

Table 2.1. Material models available for BWR analyses.

\begin{tabular}{c|l|l}
\hline Property & Description & BISON source code \\
\hline $\boldsymbol{U}_{2}-\boldsymbol{G d}_{2} \boldsymbol{O}_{3}$ properties & \\
\hline 2.1 & thermal conductivity up to 60MWd/kgU & ThermalFuel \\
\hline 2.2 & thermal conductivity at extended burnup & ThermalFuel \\
\hline 2.3 & specific heat capacity & ThermalFuel \\
$\boldsymbol{P}$ ure zirconium properties & \\
\hline 2.4 & thermal conductivity & ZrThermal \\
\hline 2.5 & specific heat capacity & ZrThermal \\
\hline 2.6 & thermal expansion eigenstrain & ZrThermalExpansionEigenstrain \\
Zirconium tin properties & \\
\hline 2.7 & thermal creep & ZrSnCreepUpdate \\
Zircaloy-2 & properties & \\
\hline 2.8 & thermal conductivity & ThermalZry \\
\hline 2.9 & specific heat capacity & ThermalZry \\
\hline 2.10 & elastic properties & ZryElasticityTensor \\
\hline 2.11 & thermal expansion & ZryThermalExpansionEigenstrain \\
\hline 2.12 & thermal and irradiation creep & ZryCreepLOCAErbacherLimbackHoppeUpdate \\
\hline 2.13 & irradiation growth & ZryIrradiationGrowthEigenstrain \\
\hline
\end{tabular}




\section{$2.1 \quad \mathrm{UO}_{2}-\mathrm{Gd}_{2} \mathrm{O}_{3}$ Properties}

\section{Thermal Properties}

This section covers newly implemented/updated thermal modeling options in BISON under three main categories: Property 2.1 for thermal conductivity models that are valid up to $60 \mathrm{MWd} / \mathrm{kgU}$; Property 2.2 for thermal conductivity models at extended burnups; and Property 2.3 for the specific heat capacity.

\section{Property 2.1: $\mathrm{UO}_{2}-\mathrm{Gd}_{2} \mathrm{O}_{3}$ fuel, thermal conductivity up to $60 \mathrm{MWd} / \mathrm{kgU}$ (ThermalFuel)}

A new thermal conductivity model is developed using an extensive set of experimental data [8], referred to as the Toptan model in the code. The model form is expressed in terms of $T$ is the fuel temperature $\left({ }^{\circ} \mathrm{C}\right), G d$ is the fraction of the gadolinia content (weight fraction), and $B u$ is the burnup (MWd/kgU), which is given by

$$
\begin{gathered}
k_{95}=k_{p h}+k_{e l} \\
k_{p h}=\frac{1}{0.1149+2.2748 \times 10^{-4} T+1.2027 g d+2.3655 \times 10^{-3} B u+\frac{6.7927 \times 10^{-3} B u^{0.28}(1-0.9 \exp (-0.04 B u))}{1+396 \exp \left(-\frac{6380}{T}\right)}}, \\
k_{e l}=7.5620 \times 10^{-3} \exp \left(2.0081 \times 10^{-3} T\right),
\end{gathered}
$$

The thermal conductivity normalized to a given porosity $p$ (unitless) [9] is obtained by

$$
k=k_{100}\left(\frac{1.0-p}{1.0+\beta p}\right)
$$

where $k_{100}$ is the thermal conductivity of the non-porous material, and $\beta(=0.5)$ is the geometrical factor [10]. Eq. 2.2 can be applied to $k_{95}$ by simply inserting $k_{100}=1.07895 k_{95}$ into the above relation. Existing literature thermal conductivity models that account for the gadolinia addition are tabulated in Table 2.2. These models exist in BISON prior to this project. A detailed review of this modeling was performed to confirm that they are implemented in the code properly.

\section{Property 2.2: $\mathrm{UO}_{2}-\mathrm{Gd}_{2} \mathrm{O}_{3}$ fuel, thermal conductivity at extended burnup (ThermalFuel)}

The thermal conductivity, $k(\mathrm{~W} / \mathrm{m} / \mathrm{K})$ at $95 \%$ theoretical density (TD) (i.e., normalized to 5 vol.\% as-fabricated porosity) is given by $[11,12]$ in the following form:

$$
k_{95}=\frac{1}{A\left(T_{i r r}, T_{a n n}, b u\right)+B\left(T_{i r r}, T_{a n n}, b u\right) T}
$$

where $b u$ is the burnup $(\mathrm{GWd} / \mathrm{t}), T_{i r r}$ is the irradiation temperature $(\mathrm{K}), T_{a n n}$ is the maximum temperature reached during out-of-pile annealing $(\mathrm{K})$, and $T$ is the instant application temperature 
(K). $A$ and $B$ are the empirical coefficients which are formulated as follows:

- Coefficient $A\left(T_{i r r}, T_{a n n}, b u\right)$. [11] defines the coefficient $A$ as:

$$
A\left(T_{i r r}, T_{a n n}, b u\right)=\delta A+\Gamma(b u, G I S)+0.046
$$

Note that [12] replaces 0.046 with 0.092623 for non-doped fuel $(g d=0)$ and $0.0524+0.3079 \times$ $10^{-2} g d+12.2031 \times 10^{-4} g d^{2}$ for the non-zero gadolinia additive, where $g d$ is the gadolinia content in wt.\%.

The effect of the irradiation defects on $A$ is defined in terms of the out-pile self-irradiation effect, $\delta A_{\text {Self }}$ and the effective concentration of irradiation defects at end of life (EOL), $\delta A_{E O L}$ as

$$
\delta A=\delta A_{\text {Self }}\left(T_{m}, b u\right)+\delta A_{E O L}\left(T_{m}, b u\right)
$$

where $T_{m}$ is the maximum temperature of $T_{i r r}$ and $T_{a n n}$ (i.e., $T_{m}=\max \left(T_{i r r}, T_{a n n}\right)$ ).

$$
\begin{gathered}
\delta A_{\text {Self }}\left(T_{m}, b u\right)= \begin{cases}0.02 F(b u) & \text { if } T_{m} \leq 900 K \\
0.02 F(b u) \frac{1450-T_{m}}{1450-900} & \text { if } 1450 K>T_{m}>900 K \\
0 & \text { if } T_{m} \geq 1450 K .\end{cases} \\
\delta A_{E O L}\left(T_{m}, b u\right)=\frac{b u}{850}\left[\frac{1}{1+\exp \left(\frac{T_{m}-950}{25}\right)}+\frac{1}{1+\exp \left(\frac{T_{m}-1300}{35}\right)}-0.0525\right] \\
F(b u)=\frac{1}{1+\exp \left(\frac{20-b u}{6}\right)}-0.015267
\end{gathered}
$$

In [12], 0.015267 is replaced with 0.03444 in their formulation.

The contribution of nonvolatile and volatile fission products is incorporated to the thermal conductivity model by $[11,12]$ as:

$$
\Gamma(b u, G I S)=9.02 \times 10^{-4} b u G I S+1.74 \times 10^{-3} b u+7.51 \times 10^{-3}
$$

where GIS is the fraction of volatile fission products present as dispersed atoms in the fuel matrix and is expressed by

$$
G I S=\frac{1-0.9 I R I M}{\left[1+\exp \left(\frac{T_{i r r}-1350}{200}\right)\right]\left[1+\exp \left(\frac{T_{a n n}-1350}{200}\right)\right]}
$$

in terms of

$$
I R I M=\frac{1}{\left[1+\exp \left(\frac{T_{i r r}-950}{30}\right)\right]\left[1+\exp \left(\frac{73-b u}{2}\right)\right]} .
$$


- Coefficient $B\left(T_{i r r}, T_{a n n}, b u\right)$. [11] defines the coefficient $B$ as:

$$
\begin{gathered}
B\left(T_{i r r}, T_{a n n}, b u\right)=B_{0}+\left(B_{1}-B_{0}\right)\left(1-\frac{\delta B}{6.5 \times 10^{-5}}\right) \\
B_{0}=-1.65 \times 10^{-6} b u+2.55 \times 10^{-4}-3.6 \times 10^{-5} I R I M \\
B_{1}=4.2 \times 10^{-7} b u+2.75 \times 10^{-4}
\end{gathered}
$$

In [12], $2.75 \times 10^{-4}$ is replaced with $2.217 \times 10^{-4}$ for non-doped fuel $(G d=0)$ and, for a non-zero gadolinia additive, $2.75 \times 10^{-4}$ is replaced with $2.553 \times 10^{-4}+8.606 \times 10^{-6} g d-0.0154 \times 10^{-4} g d^{2}$ where $g d$ is the gadolinia content in wt.\%.

$$
\begin{gathered}
\delta B=F(b u) \delta B_{E O L}\left(T_{m}, b u\right) \\
\delta B_{E O L}\left(T_{m}, b u\right)=\frac{b u}{34}\left[\frac{4.0 \times 10^{-5}}{1+\exp \left(\frac{T_{m}-950}{25}\right)}+\frac{2.5 \times 10^{-5}}{1+\exp \left(\frac{T_{m}-1300}{35}\right)}\right]
\end{gathered}
$$

To ensure that the model (2.2) is implemented in BISON correctly and outputs in reasonable values, we create the thermal conductivity plots. Fig. 2.1 shows the model comparison from each model at various temperatures. The models differentiate from each other slightly for the non-doped uranium dioxide fuel.

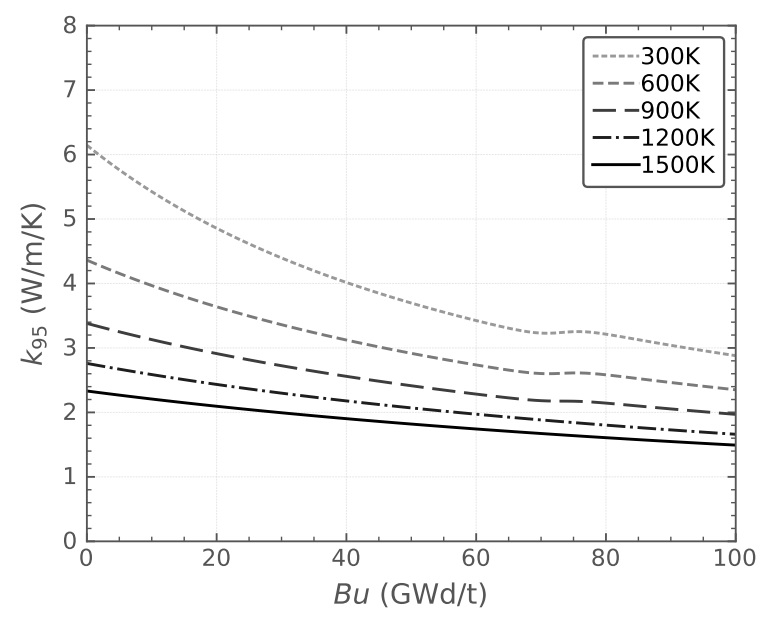

(a) The Staicu model $(G d=0)$.

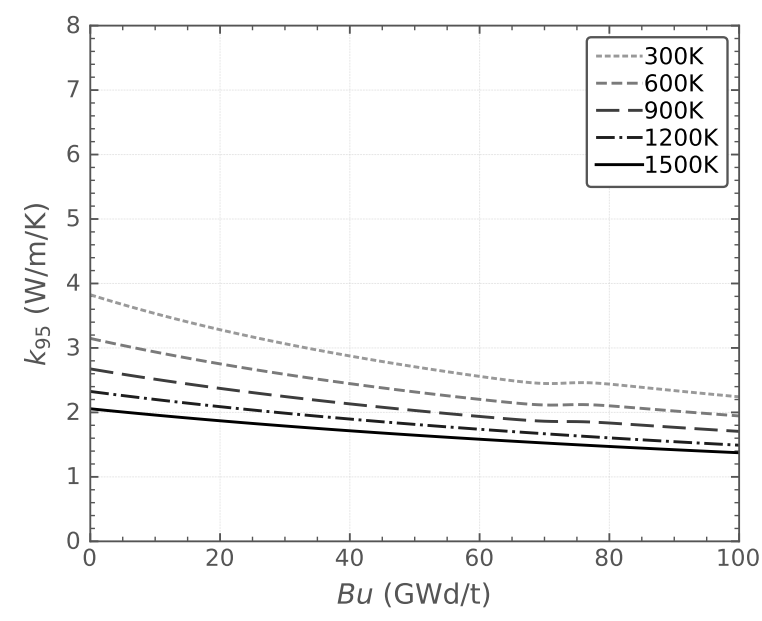

(b) The Staicu model $(G d=10 \mathrm{wt} \%)$.

Figure 2.1. Comparison of the thermal conductivity model at $T=T_{i r r}$ using (a) the Ronchi model, (b) the Staicu model, and (c) the Staicu model with an arbitrary gadolinia concentration of 10wt.\%. The thermal conductivity increases in the transition region where the HBS commences to form. [13] attributes the increase in the thermal conductivity in the HBS formation to the decrease in the fission gas concentration dissolved in the matrix. The degradation of the thermal conductivity resumes as a result of the accumulation of fission products for further increase of the burnup. 
Table 2.2. A list of the conventional Gd-doped $\mathrm{UO}_{2}$ thermal conductivity models in BISON. The formulation of each model is provided along with the reported applicability range. Maxwell's relation (Eq. 2.2) is applied for the porosity correction unless it is reported differently. The units of the state variables: $k(\mathrm{~W} / \mathrm{m}-\mathrm{K})$, $T_{K}(\mathrm{~K}), T_{C}\left({ }^{\circ} \mathrm{C}\right), p(-), B u(\mathrm{MWd} / \mathrm{kgU}), B(\mathrm{MWd} / \mathrm{kg}$ urania), $\beta($ at.\%), and $g d($ weight fraction).

\begin{tabular}{|c|c|}
\hline The Halden model $[14$, p.3.4] & Applicability \\
\hline $\begin{array}{l}k_{95}=k_{p h}+k_{e l} \\
k_{p h}=\frac{1}{0.1148+1.1599(g d+x)+0.0040 B+2.475 \times 10^{-4}(1-0.00333 B) \min \left(1650, T_{c}\right)} \\
k_{e l}=0.0132 \exp \left(0.00188 T_{c}\right) \\
\quad \text { The modified NFI model }[14]\end{array}$ & $\begin{array}{r}T \text { in }[300 \mathrm{~K}, 3000 \mathrm{~K}] \\
B u \text { in }[0,62 \mathrm{GWd} / \mathrm{MTU}] \\
P u \text { in }[0,7 \mathrm{wt} \%] \\
T D \text { in }[92 \%, 97 \%]\end{array}$ \\
\hline $\begin{array}{l}k_{95}=k_{p h}+k_{e l}, \\
k_{p h}=\frac{1}{0.0452+1.1599 g d+2.46 \times 10^{-4} T+0.00187 B u+\frac{0.038 B u 0.28[1-0.9 \exp (-0.04 B u)])}{(1+396 \exp (-6380 / T))},} \\
k_{e l}=\frac{3.5 \times 10^{9}}{T^{2}} \exp \left(-\frac{16361}{T}\right) \quad \text { The NFIR model }[15,16]\end{array}$ & $\begin{array}{r}T \text { in }[300 \mathrm{~K}, 3000 \mathrm{~K}] \\
B u \text { in }[0,62 \mathrm{GWd} / \mathrm{MTU}] \\
T D \text { in }[92 \%, 97 \%] \\
G d \text { in }[0,10 \mathrm{wt} \%]\end{array}$ \\
\hline $\begin{array}{l}k_{95}=(1-F) k_{\text {start }}+F k_{\text {end }}+k_{e l} \\
F=0.5\left(1+\tanh \left(\frac{T_{c}-900}{150}\right)\right), \\
k_{\text {start }}=\frac{1}{\text { term }_{0}+6.14 \times 10^{-3} B u-1.4 \times 10^{-5} B u^{2}+\left\{2.5 \times 10^{-4} \exp (-1.268763 g d)-1.81 \times 10^{-6} B u\right\} T_{c}}, \\
k_{\text {end }}=\frac{1}{\text { term } m_{0}+2.6 \times 10^{-3} B u+\left\{2.5 \times 10^{-4} \exp (-1.268763 g d)-2.7 \times 10^{-7} B u\right\} T_{c}} \\
k_{e l}=0.0132 \exp \left(0.00188 T_{c}\right), \\
\text { term }_{0}=\left\{\begin{array}{c}9.592 \times 10^{-2}, \quad g d=0 \\
g d i r \cdot g d f a c, \quad g d \neq 0\end{array}\right. \\
g d i r=0.1197 \tanh \left(1 \times 10^{2} g d\right)^{0.1}+1.214167 g d+5.40625 g d^{2}-51.82292 g d^{3} \\
g d f a c=0.65227273+2.7273 g d-2.25 g d \tanh \left(1 \times 10^{-5} B u\right) \\
\text { The porosity correction multiplier is: }\left(\frac{1.0-p\left[2.58-5.8 \times 10^{-4} T_{C}\right]}{1.0-0.05\left[2.58-5.8 \times 10^{-4} T_{C}\right]}\right)\end{array}$ & \\
\hline
\end{tabular}




\section{Property 2.3: $\mathrm{UO}_{2}-\mathrm{Gd}_{2} \mathrm{O}_{3}$ fuel, specific heat capacity (ThermalFuel)}

The specific heat capacity $(\mathrm{J} / \mathrm{kg} / \mathrm{K})$ is expressed by:

$$
c_{P}=\frac{K_{1} \theta^{2} \exp \left(\frac{\theta}{T}\right)}{T^{2}\left[\exp \left(\frac{\theta}{T}\right)-1\right]^{2}}+K_{2} T+\frac{Y K_{3} E_{D}}{2 R T^{2}} \exp \left(-\frac{E_{D}}{R T}\right)
$$

where $T$ is the temperature $(\mathrm{K}), Y$ is the oxygen-to-metal ratio (-), and $R$ is the universal gas constant $(8.3143 \mathrm{~J} / \mathrm{mol} / \mathrm{K})$. The empirical coefficients $\left(K_{1}, K_{2}, K_{3}, \theta\right.$, and $\left.E_{D}\right)$ are tabulated in Table 2.3 for $\mathrm{UO}_{2}$ and $\mathrm{GdO}_{2}$. The specific heat capacity of $G d$-doped $\mathrm{UO}_{2}$ fuels (see Fig. 2.2) is computed as:

$$
c_{P}=(1-G d) c_{P, U O_{2}}+G d c_{P, G d O_{2}}
$$

in terms of the fraction of gadolina content, $G d(-)$, and the specific heat capacity, $c_{P, i}$ for $i=\mathrm{UO}_{2}$ and $\mathrm{GdO}_{2}$.

Table 2.3. The empirical constants used in Eq. 2.17 from [17].

\begin{tabular}{l|c|c|c|c|c}
\hline & $K_{1}(\mathrm{~J} / \mathrm{kg} / \mathrm{K})$ & $K_{2}\left(\mathrm{~J} / \mathrm{kg} / \mathrm{K}^{2}\right)$ & $K_{3}(\mathrm{~J} / \mathrm{kg})$ & $\theta(\mathrm{K})$ & $E_{D}(\mathrm{~J} / \mathrm{mol})$ \\
\hline $\mathrm{UO}_{2}$ & 296.70 & $2.430 \times 10^{-2}$ & $8.745 \times 10^{7}$ & 535.285 & $1.577 \times 10^{5}$ \\
$\mathrm{GdO}_{2}$ & 315.86 & $4.044 \times 10^{-2}$ & 0.0 & 348.000 & 0.0 \\
\hline
\end{tabular}

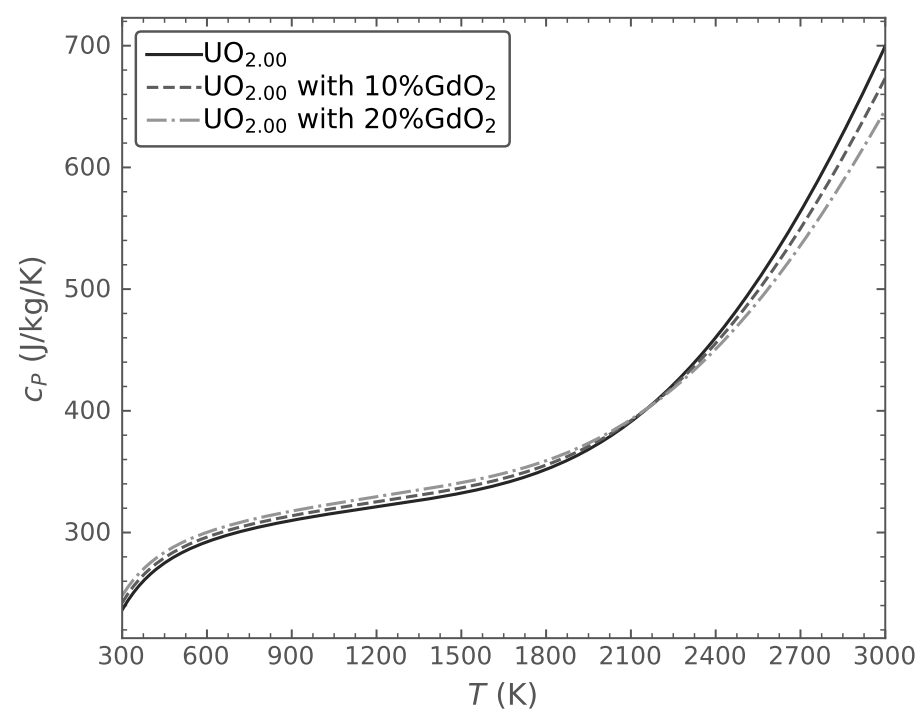

Figure 2.2. Specific heat capacity of $\mathrm{UO}_{2}$ at arbitrarily chosen gadolinia concentrations (0 to 20 wt. $\%$ ). 


\section{$2.2 \quad$ Pure Zirconium Properties}

Pure zirconium is one material used for liners in BWRs. For nuclear applications, the majority material property correlations are for the zirconium alloys Zircaloy-2 and Zircaloy-4. In BWRs, the cladding material is Zircaloy-2; thus, for material properties for which a pure zirconium model is unavailable, those of Zircaloy-2 (see Section 2.4) are used.

\section{Thermal Properties}

Property 2.4: Pure zirconium, thermal conductivity (ZrThermal)

The thermal conductivity $(\mathrm{W} / \mathrm{m}-\mathrm{K})$ of pure zirconium is given by [18]:

$$
k=8.8527+7.0820 \times 10^{-3} T+2.5329 \times 10^{-6} T^{2}+\frac{2.9918 \times 10^{3}}{T}
$$

where $T$ is the temperature (in $\mathrm{K}$ ) and is valid in $[298 \mathrm{~K}, 2000 \mathrm{~K}]$.

\section{Property 2.5: Pure zirconium, specific heat capacity (ZrThermal)}

The specific heat correlation depends upon whether the zirconium is in the $\alpha$-phase $(298 \leq \mathrm{T} \leq 1139$ $\mathrm{K})$ or $\beta$-phase $(1139<\mathrm{T} \leq 2128 \mathrm{~K})[18]$.

$$
c_{P}= \begin{cases}24.1618+8.75582 \times 10^{-3} T-\frac{69942}{T^{2}} & \alpha \text {-phase } \\ 25.607406+6.80168 \times 10^{-4} T+5.837384 \times 10^{-8} T^{2}+9.13714728 \times 10^{-10} T^{3}-\frac{50466}{T^{2}} & \beta \text {-phase }\end{cases}
$$

where $T$ is the temperature (in $\mathrm{K}$ ).

\section{Mechanical Properties}

Property 2.6: Pure zirconium, thermal expansion (ZrThermalExpansionEigenstrain)

This material calculates the temperature dependent thermal expansion of $\mathrm{Zr}$ using a correlation from [18]. The thermal expansion $(\Delta L / L)$ is written in terms of temperature, $T$ (in $\mathrm{K}$ ):

$$
\frac{\Delta L}{L}= \begin{cases}-1.11 \times 10^{-3}+2.325 \times 10^{-6} T+5.595 \times 10^{-9} T^{2}-1.768 \times 10^{-12} T^{3} & \alpha \text {-phase } \\ -7.59 \times 10^{3}+1.474 \times 10^{-6} T-5.140 \times 10^{-9} T^{2}+1.559 \times 10^{-12} T^{3} & \beta \text {-phase }\end{cases}
$$




\subsection{Zirconium Tin Properties}

Low tin content $(<1 \%)$ zirconium-tin alloys are another option used for BWR liners. As with pure zirconium, models for low tin content alloys for nuclear applications is scarce; therefore, in the absence of a specific model, those of Zircaloy-2 (see Section 2.4) are used.

\section{Mechanical Properties}

Property 2.7: Zirconium tin, thermal creep (ZrSnCreepUpdate)

The creep rate is a given by [19]:

$$
\dot{\epsilon}=A[\sinh (\alpha \sigma)]^{n} \exp \left(-\frac{Q}{R T}\right)
$$

where $A\left(\mathrm{~s}^{-1}\right), \alpha\left(\mathrm{MPa}^{-1}\right), n(-)$, and $Q(\mathrm{~kJ} / \mathrm{mol})$ are material parameters, $R(\mathrm{~kJ} / \mathrm{mol}-\mathrm{K})$ is the ideal gas constant, and $T(\mathrm{~K})$ is the temperature. The material constants are are all fourth order polynomials of the inelastic strain, $\epsilon$, as follows:

$$
\ln (A)=\sum_{i=0}^{4} A_{i} \epsilon^{i}, \quad \alpha=\sum_{i=0}^{4} B_{i} \epsilon^{i}, \quad n=\sum_{i=0}^{4} C_{i} \epsilon^{i}, \quad \text { and } \quad Q=\sum_{i=0}^{4} D_{i} \epsilon^{i}
$$

where $A_{i}$ through $D_{i}$ are the coefficients, which are provided in Table 2.4.

Table 2.4. Coefficients used in the polynomial fits for the material constants used in the Zr- $0.3 \% \mathrm{Sn}$ creep correlation.

\begin{tabular}{c|c|c|c|c|c}
\hline & $i=0$ & $i=1$ & $i=2$ & $i=3$ & $i=4$ \\
\hline$A_{i}$ & 53.365 & -39.774 & -40.405 & 287.21 & -264.92 \\
\hline$B_{i}$ & 0.0347 & -0.2091 & 0.817 & -1.3775 & 0.85420 \\
\hline$C_{i}$ & 3.6433 & 40.123 & -201.32 & 373.51 & -242.19 \\
\hline$D_{i}$ & 506.22 & -730.03 & 1345.3 & -466.67 & -433.33 \\
\hline
\end{tabular}




\subsection{Zircaloy-2 Properties}

Zircaloy-2 is the cladding material of choice in BWRs. This section outlines the material models available in BISON for Zircaloy-2. As described in previously, the material models (except irradiation growth) described in this section are also applied to liners when a specific liner material does not exist. The cold work of Zircaloy-2 is taken as $0 \%$ [17].

\section{Thermal Properties}

Property 2.8: Zircaloy-2, thermal conductivity (ThermalZry)

The thermal conductivity is given by

$$
k=7.668 \times 10^{-8} T^{3}-1.450 \times 10^{-5} T^{2}+2.088 \times 10^{-2} T+7.511
$$

where $T$ is the temperature (in $\mathrm{K}$ ).

\section{Property 2.9: Zircaloy-2, specific heat capacity (ThermalZry)}

The specific heat is also obtained from MATPRO and is a piecewise linear function of the tabulated values in Table 2.5. For values above $1248 \mathrm{~K}$ the specific heat is set to $356 \mathrm{~J} / \mathrm{kg} / \mathrm{K}$.

Table 2.5. Specific heat capacities for Zircaloy.

\begin{tabular}{c|c|c|c}
\hline$T(\mathrm{~K})$ & $c_{P}(\mathrm{~J} / \mathrm{kg} / \mathrm{K})$ & $T(\mathrm{~K})$ & $c_{P}(\mathrm{~J} / \mathrm{kg} / \mathrm{K})$ \\
\hline 300 & 281 & 1153 & 719 \\
\hline 400 & 302 & 1173 & 816 \\
\hline 640 & 331 & 1193 & 770 \\
\hline 1090 & 375 & 1213 & 619 \\
\hline 1093 & 502 & 1233 & 469 \\
\hline 1113 & 590 & 1248 & 356 \\
\hline 1133 & 615 & & \\
\hline
\end{tabular}




\section{Mechanical Properties}

Property 2.10: Zircaloy-2, elastic constants (ZryElasticityTensor)

The Young's modulus in the $\alpha$ and $\beta$ phases is calculated by:

$$
E=\left\{\begin{array}{cc}
\frac{\left(1.088 \times 10^{11}-5.475 \times 10^{7} T+\left(6.61 \times 10^{11}+5.912 \times 10^{8} T\right) \Delta\right)}{0.88+0.12 \exp \left(-\frac{\phi}{1 \times 10^{25}}\right)} & \alpha \text {-phase } \\
9.21 \times 10^{10}-4.05 \times 10^{7} T & \beta \text {-phase }
\end{array}\right.
$$

and the shear modulus is

$$
G=\left\{\begin{array}{cc}
\frac{4.04 \times 10^{10}-2.168 \times 10^{7} T+\left(7.07 \times 10^{11}-2.315 \times 10^{8} T\right) \Delta}{0.88+0.12 \exp \left(-\frac{\phi}{1 \times 10^{25}}\right)} & \alpha \text {-phase } \\
3.49 \times 10^{10}-1.66 \times 10^{7} T & \beta \text {-phase }
\end{array}\right.
$$

where $T(\mathrm{~K})$ is the temperature, $\Delta$ is the average oxygen concentration minus the oxygen concentration of the as-received alloy, and $\phi\left(n / m^{2}\right)$ is the fast neutron fluence. It should be noted that there is a cold work contribution to the general equations for $E$ and $G$, which have been ommitted here because the cold work for Zircaloy-2 is $0 \%$. The Poisson's ratio, $\nu$ is computed via the following relation:

$$
\nu=\frac{E}{2 G}-1
$$

\section{Property 2.11: Zircaloy-2, thermal expansion (ZryThermalExpansionEigenstrain)}

The thermal expansion of these materials is anisotropic with different strains forming in the diametrical and axial directions. The diametrical and axial thermal strains, $\epsilon_{\text {dia }}$ and $\epsilon_{\text {axial }}$, are given by MATPRO in the $\alpha$-phase $(T<1073 \mathrm{~K})$ and $\beta$-phase $(T>1244 \mathrm{~K})$ :

$$
\begin{gathered}
\epsilon_{\text {axial }}=\left\{\begin{array}{cc}
-2.5060 \times 10^{-5}+4.441 \times 10^{-6} T_{C} & \alpha \text {-phase } \\
-8.3 \times 10^{-3}+9.70 \times 10^{-6} T_{C} & \beta \text {-phase }
\end{array}\right. \\
\epsilon_{\text {dia }}=\left\{\begin{array}{cc}
-2.373 \times 10^{-4}+6.721 \times 10^{-6} T_{C} & \alpha \text {-phase } \\
-6.8 \times 10^{-3}+9.70 \times 10^{-6} T_{C} & \beta \text {-phase }
\end{array}\right.
\end{gathered}
$$

The linear interpolation is employed between the $\alpha$ and $\beta$-phase values is used in the $\alpha+\beta$-phase $(1073 \mathrm{~K} \leq T \leq 1244 \mathrm{~K})$. 
Property 2.12: Zircaloy-2, creep (ZryCreepLOCAErbacherLimbackHoppeUpdate)

Low temperature thermal creep of Zircaloy-2 is given by the Limbäck-Andersson [20] model:

$$
\begin{aligned}
\dot{\epsilon}_{t h \_l o w} & =5.47 \times 10^{8} \frac{E}{T}\left(\sinh \left(\frac{a_{i} \sigma_{\text {eff }}}{E}\right)\right)^{3} .5 \exp \left(-\frac{198000}{R T}\right) \\
a_{i} & =650\left[1.0-0.56\left(1-e^{\left(-1.4 \times 10^{-24} \Phi^{1.3}\right)}\right)\right] \\
E & =1.148 \times 10^{5}-59.9 T
\end{aligned}
$$

where $\dot{\epsilon}_{\text {th_low }}$ is the low temperature thermal creep rate $\left(\mathrm{hr}^{-1}\right), E$ is the Young's modulus (MPa), $T$ is the temperature $(\mathrm{K}), P h i$ is the fast neutron fluence $\left(\mathrm{n} / \mathrm{cm}^{2}\right), \sigma_{\text {eff }}$ is the effective (Von Mises) stress (MPa), and $R$ is the ideal gas constant $(8.314 \mathrm{~J} / \mathrm{mol}-\mathrm{K})$. High temperature $(>900 \mathrm{~K})$ thermal creep of the Zircaloy-2 is of the form [21]:

$$
\dot{\epsilon}_{t h \_h i g h}=A \sigma_{e f f}^{n} \exp \left(-\frac{Q}{R T}\right)
$$

where $\dot{\epsilon}_{t h \_h i g h}$ is the high temperature creep rate $\left(\mathrm{s}^{-1}\right), A$ is the creep coefficient, $\sigma_{\text {eff } f}$ is the effective (Von Mises) stress, $n$ is the stress exponent, and $Q$ is the activation energy. These parameters depend upon the phase of the alloy, as summarized in Table 2.6, where the activation energy in the $\alpha$-phase is given by:

$$
A=3.21 \times 10^{5}+24.69(T-923.15)
$$

Linear interpolation is used for mixed phases not equal to $50 \% \alpha-50 \% \beta$ for $\epsilon \leq 3 \times 10^{-3}$. For $\epsilon>$ $3 \times 10^{-3}$ linear interpolation is used for $Q$ and $n$ and linear interpolation of $\ln (A)$ is used for $A$. Between the low temperature creep model upper limit of $>700 \mathrm{~K}$ and the lower limit of $>900 \mathrm{~K}$ for the high temperature creep model interpolation between the two correlations are used.

The irradiation creep law used for Zircaloy-2 (with a recrystallized annealed microstructure) is given by:

$$
\dot{\epsilon}_{i r r}=1.654 \times 10^{-24} \phi^{0.85} \sigma_{e f f}
$$

where $\dot{\epsilon}_{i r r}$ is the irradiation creep rate $\left(\mathrm{hr}^{-1}\right), \phi$ is the fast neutron flux $\left(\mathrm{n} / \mathrm{m}^{2}-\mathrm{s}\right)$, and $\sigma_{e f f}$ is the effective stress (MPa). 


\section{Property 2.13: Zircaloy-2, irradiation growth (ZryIrradiationGrowthEigenstrain)}

Zircaloy-2 undergoes axial growth and subsequent diametrical shrinkage due to irradiation. The correlation of irradiation growth is given by:

$$
\epsilon_{\text {irr }}=1.09 \times 10^{-21} \Phi^{0.845}
$$

where $\Phi$ is the fast neutron fluence $\left(\mathrm{n} / \mathrm{m}^{2}\right)$.

Table 2.6. Parameters for high temperature creep of Zircaloy-2.

\begin{tabular}{c|c|c|c}
\hline Phase & $\mathrm{A}\left(\mathrm{MPa}^{n} / \mathrm{s}\right)$ & $\mathrm{Q}(\mathrm{kJ} / \mathrm{mol})$ & $\mathrm{n}(-)$ \\
\hline$\alpha$ & 8737 & See Eq. 2.32 & 5.89 \\
\hline $50 \% \alpha-50 \% \beta$ & 0.24 & $1.02355 \times 10^{5}$ & 2.33 \\
\hline$\beta$ & 7.9 & $1.41919 \times 10^{5}$ & 3.78 \\
\hline
\end{tabular}

\subsection{Hydrogen Migration/Redistribution Model of BISON}

The use of different alloying materials, such as the fuel liners (e.g., pure zirconium or zirconium tin), to increase the margin to PCMI failure, enabling more flexible operation. Such liners tend to exhibit much higher hydrogen contents than the rest of the cladding (in the hottest side of the cladding wall) ${ }^{1}$. The concentration of hydrogen and consequent localized precipitation of hydrides can cause the failure of the cladding at small strains. Our task for this report is to document the BISON's existing model for the hydrogen migration and redistribution in the cladding.

Background The hydride migration and redistribution is schematically illustrated in Fig. 2.3. Hydrogen is picked/absorbed by the cladding from the chemical reaction occurring at the outer surface of the cladding with the water (i.e., oxidation). Later, hydrogen is transported in the cladding via two main diffusion mechanisms in which driving forces are temperature and concentration gradients that apply in opposite directions: Soret effect and Fickian diffusion, respectively. Depending upon the applied stress, the orientation of hydride precipitation will differentiate.

Previous studies on the hydrogen migration and redistribution in BISON are performed and/or implemented by several researchers $[24,25,26,27,28]$. Herein, we provide a brief summary of up-to-date model (available) in the code - referred to as the Hydride Nucleation - Growth - Dissolution Model (HNGD) - is

\footnotetext{
${ }^{1}$ Experimental evidence can be found in the literature, particularly for the liner-cladding geometry in [22, 23].
} 
given by the following formation:

$$
\begin{aligned}
\frac{\partial T}{\partial t} & =\alpha \nabla^{2} T \\
\frac{\partial C_{P r e c}}{\partial t} & =S \\
\frac{\partial C_{s s}}{\partial t} & =-\nabla\left(-D \nabla C_{s s}-\frac{D Q^{\star} C_{s s}}{R T^{2}} \delta T\right)-S
\end{aligned}
$$

where $T$ is temperature, $\alpha$ is thermal diffusivity, $C_{\text {prec }}$ is the concentration of precipitated hydrogen as hydrides, $C_{s s}$ is the concentration of dissolved hydrogen in the solid solution, $Q^{\star}$ is the heat of transport, and $R$ is the universal gas constant $(8.3143 \mathrm{~J} / \mathrm{mol} / \mathrm{K})$. The diffusion coefficient is computed according to the Arrhenius law: $D=D_{0} \exp \left(-E_{D} / R T\right)$ in terms of $D_{0}$ is the constant and $E_{D}$ is the activation energy.

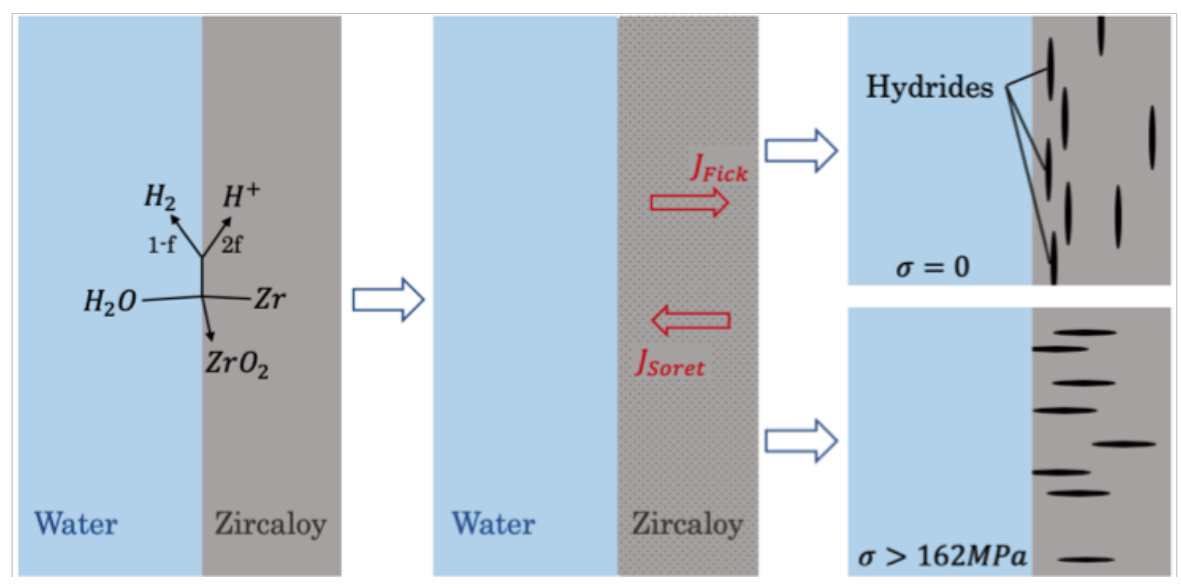

Figure 2.3. Schematic illustration of hydride migration and redistribution within a Zircaloy cladding, from [27]. Process can be categorized under three sequential categories: (a) hydrogen is absorbed by the cladding from the chemical reaction occuring at the outer surface of the cladding with the water, i.e., oxidation via $\mathrm{Zr}+2 \mathrm{H}_{2} \mathrm{O} \rightarrow \mathrm{ZrO}_{2}+2 \mathrm{H}_{2}$ (left figure); (b) hydrogen is transported in the cladding via two main diffusion mechanisms in which driving forces are temperature (Soret effect) and concentration gradients (Fick's law) (middle figure); and (c) hydrogen is either dissolved in the solid solution or precipitates as hydrides whose orientation is heavily dependent on the applied stress (right figure).

Instructions Details of the HNGD modeling in BISON are described in Algorithms 1 through 3. Output parameters from this model are the concentration of dissolved hydrogen, $C_{s s}$, and concentration of hydrogen as hydride, $C_{\text {prec }}$, in addition to the temperature, $T$, from the coupled heat conduction equation. There are several input parameters - optional parameters with preset default values for the typical Zircaloy cladding - for this model, which are tabulated in Table 2.7. The hydrogen kinetics parameters differentiate for the materials of dissimilar composite clad layers. Some representative experimental values are tabulated in Table 2.8 for hydrogen diffusion kinetics and precipitation/dissolution kinetics of zirconium-hydrides. 
Considering the interface of the liner and cladding, the diffusion lengths should be sufficiently small to have accurate predictions between the dissimilar clad layers. This typically requires more refined meshes for the diffusion computations (e.g., hydrogen migration and redistribution modeling in the cladding in our case). To speed up the full-length fuel rod analyses, BISON allows a flexibility to perform a single simulation using two different mesh files at the same time: a coarser mesh for the typical thermo-mechanical fuel rod analysis and a finer mesh for the diffusion calculations. The computational results are performed on the refined mesh for the diffusion calculations and interpolated back to the coarser mesh to complete the typical fuel rod analysis. This can be quite useful in terms of having accurate and faster simulations. This approach has been previously employed by [24] for the spent fuel applications ${ }^{2}$ and is useful to investigate cladding failure with a specific hydride distribution - considering the hydride geometry and its distribution within the liner cladding - for full-length fuel rod analyses next year.
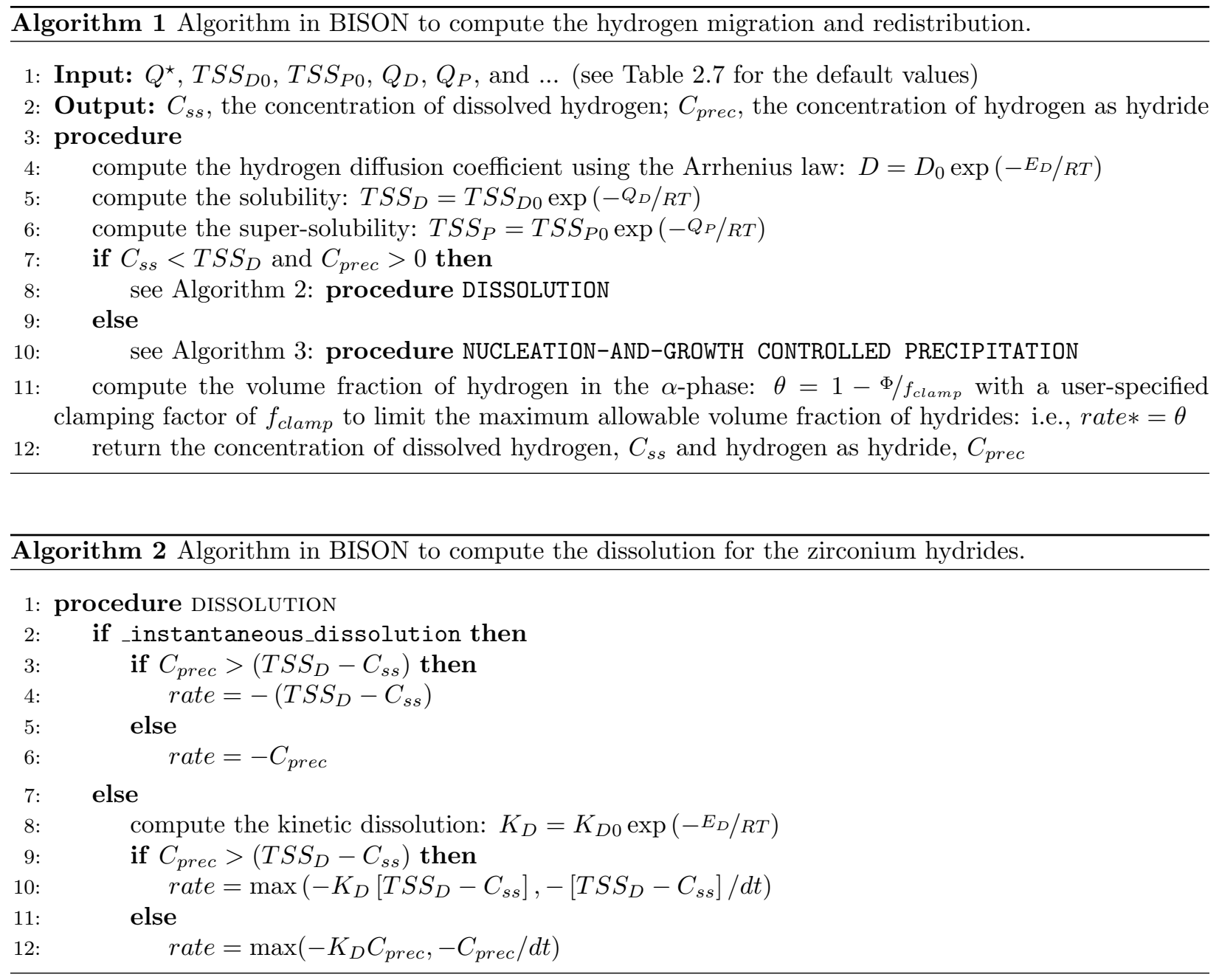

\footnotetext{
${ }^{2}$ One can find the example BISON input under bison/examples/spent_fuel/.
} 


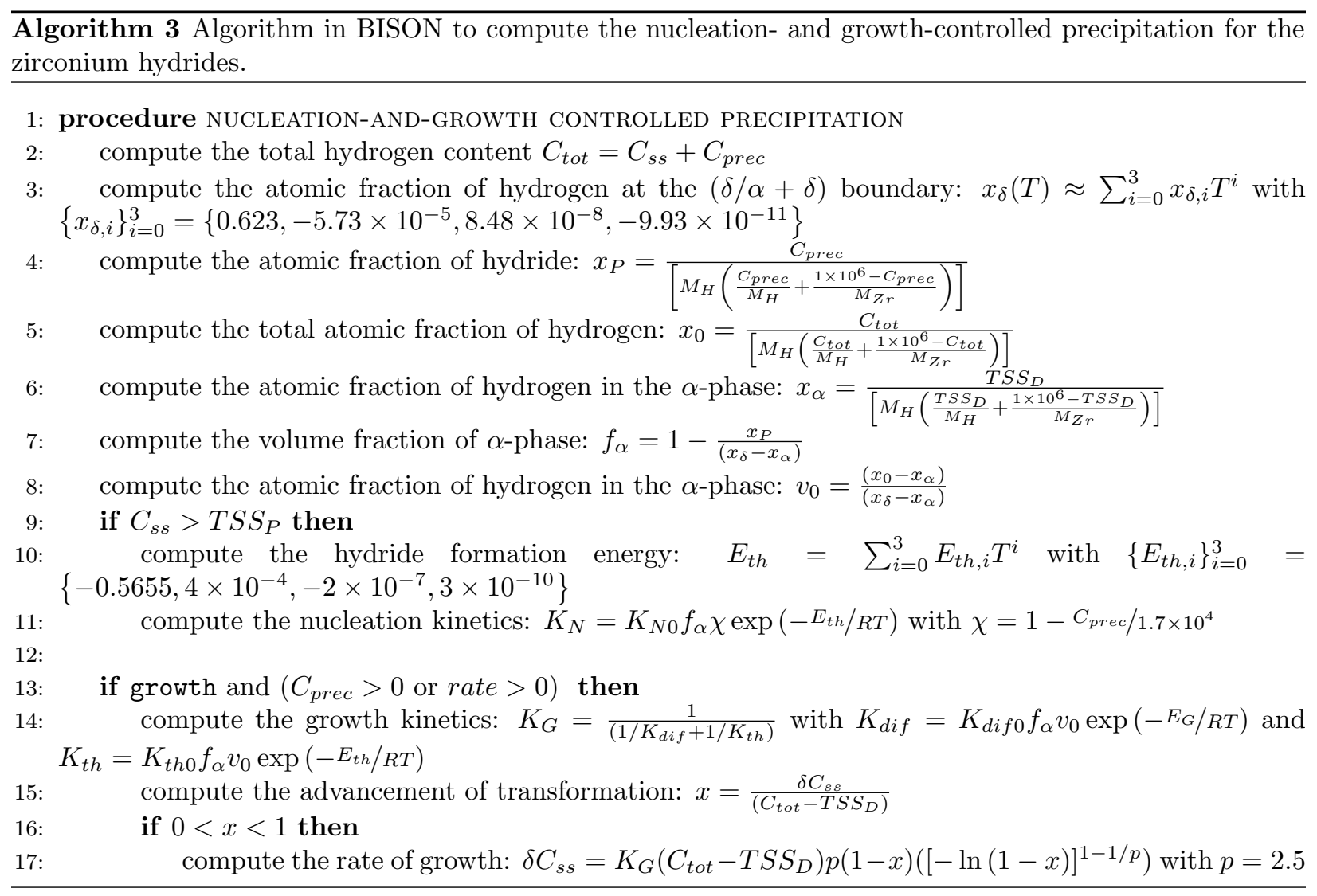

Table 2.7. BISON cladding hydrides action parameters

\begin{tabular}{|c|c|c|c|c|}
\hline Symbol & BISON input parameter & Description & Default & Unit \\
\hline$C_{\text {prec }}$ & hydrogen_as_hydride_ppm & Concentration of hydrogen as hydride & & wt.ppm \\
\hline$C_{s s}$ & hydrogen_as_solution_ppm & Concentration of dissolved hydrogen & & wt.ppm \\
\hline$T$ & temperature & Temperature & & $\mathrm{K}$ \\
\hline$D_{0}$ & diffusivity_frequency_factor & Frequency factor for Arrhenius hydrogen diffusivity & $1.08 \times 10^{-6}$ & $\mathrm{~m}^{2} / \mathrm{s}$ \\
\hline$E_{D}$ & diffusivity_activation_energy & Activation energy for Arrhenius hydrogen diffusivity & 44000 & $\mathrm{~J} / \mathrm{mol}$ \\
\hline$E_{G}$ & growth_diffusion_kinetic_energy & Activation energy for Arrhenius reaction-driven growth & 86806 & $\mathrm{~J} / \mathrm{mol}$ \\
\hline$K_{D 0}$ & dissolution_kinetic_coef & Coefficient for Arrhenius dissolution kinetics & $1.11 \times 10^{3}$ & $\mathrm{~s}^{-1}$ \\
\hline$K_{\text {dif } 0}$ & growth_reaction_kinetic_coef & Coefficient for Arrhenius diffusion-driven growth kinetics & $1.6 \times 10^{-5}$ & $\mathrm{~s}^{-1}$ \\
\hline$K_{t h 0}$ & growth_diffusion_kinetic_coef & Coefficient for Arrhenius reaction-driven growth kinetics & $5.35 \times 10^{5}$ & $\mathrm{~s}^{-1}$ \\
\hline$K_{N 0}$ & nucleation_kinetic_coef & Coefficient for Arrhenius nucleation kinetics & $2.75 \times 10^{-5}$ & $\mathrm{~s}^{-1}$ \\
\hline$Q^{\star}$ & heat_of_transport & Heat of transport for hydrogen in cladding & $2.55 \times 10^{4}$ & $\mathrm{~J} / \mathrm{mol}$ \\
\hline$Q_{D}$ & solubility_activation_energy & Activation energy for Arrhenius TSSd & 35459 & $\mathrm{~J} / \mathrm{mol}$ \\
\hline$Q_{P}$ & super_solubility_activation_energy & Activation energy for Arrhenius TSSp & 25249 & $\mathrm{~J} / \mathrm{mol}$ \\
\hline$T S S_{D 0}$ & solubility_coef & Frequency factor for Arrhenius TSSd & 101999 & wt.ppm \\
\hline$T S S_{P 0}$ & super_solubility_coef & Frequency factor for Arrhenius TSSp & 30853 & wt.ppm \\
\hline \multirow[t]{2}{*}{$f_{\text {clamp }}$} & hydride_clamp & Factor limiting the maximum hydride content in a node & 1 & \\
\hline & $\begin{array}{l}\text { instantaneous_dissolution } \\
\text { growth }\end{array}$ & Boolean to activate dissolution at equilibrium at all time & False & \\
\hline
\end{tabular}


Table 2.8. Representative experimental values taken from [25, Table 1-3].

\begin{tabular}{|c|c|c|c|c|c|c|c|c|c|}
\hline \multirow{2}{*}{\multicolumn{4}{|c|}{ Hydrogen diffusion parameters }} & \multicolumn{6}{|c|}{ Precipitation/dissolution kinetics of zirconium-hydrides } \\
\hline & & & & & \multicolumn{2}{|c|}{ Precipitation } & \multicolumn{2}{|c|}{ Dissolution } & \multirow[b]{2}{*}{ Ref } \\
\hline Material & $\begin{array}{l}D_{0} \times 10^{3} \\
\left(\mathrm{~cm}^{2} / \mathrm{s}\right)\end{array}$ & $\begin{array}{c}E_{D} \\
(\mathrm{~kJ} / \mathrm{mol})\end{array}$ & Ref. & Material & $\begin{array}{c}T S S_{D 0} \times 10^{-3} \\
\quad \text { (wt.ppm) }\end{array}$ & $\begin{array}{c}Q_{D} \\
(\mathrm{~kJ} / \mathrm{mol})\end{array}$ & $\begin{array}{c}T S S_{P 0} \times 10^{-3} \\
\quad \text { (wt.ppm) }\end{array}$ & $\begin{array}{c}Q_{P} \\
(\mathrm{~kJ} / \mathrm{mol})\end{array}$ & \\
\hline \multirow[t]{2}{*}{ Zirconium } & 7.73 & 45.3 & {$[29]$} & \multirow[t]{10}{*}{ Zirconium } & 49.0208 & 28.6604 & - & - & [30] \\
\hline & 4.15 & 39.6 & {$[31]$} & & 129.3140 & 36.1079 & - & - & {$[32]$} \\
\hline \multirow[t]{2}{*}{ Zircaloy-2 } & 5.84 & 43.1 & {$[29]$} & & 147.267 & 35.9824 & - & - & [33] \\
\hline & 2.17 & 35.1 & {$[34]$} & & 145.801 & 35.9406 & - & - & {$[35]$} \\
\hline \multirow[t]{2}{*}{ Zircaloy-4 } & 7.90 & 44.9 & {$[29]$} & & 140.084 & 36.8192 & - & - & {$[36]$} \\
\hline & 0.80 & 33.2 & {$[37]$} & & 228.662 & 39.4133 & - & - & [38] \\
\hline \multicolumn{4}{|c|}{ Heat of transport } & & 442.413 & 44.6014 & - & - & [39] \\
\hline Material & \multicolumn{2}{|c|}{$\mathrm{Q}^{\star}(\mathrm{kJ} / \mathrm{mol})$} & Ref. & & 115.844 & 35.9406 & - & - & {$[36]$} \\
\hline Zirconium & 24 & -25.1 & {$[40]$} & & 130.614 & 36.4426 & - & - & {$[36]$} \\
\hline \multirow{12}{*}{ Zircaloy-4 } & \multirow{12}{*}{30.} & -31.0 & {$[37]$} & & 52.600 & 28.068 & 141.000 & 38.104 & {$[41]$} \\
\hline & & & & Zircaloy-2 & 849.655 & 31.7984 & - & - & [34] \\
\hline & & & & & 408.399 & 41.7563 & - & - & [38] \\
\hline & & & & & 52.600 & 28.068 & 128.000 & 36.540 & {$[42]$} \\
\hline & & & & & 13.320 & 21.170 & 108.150 & 21.170 & {$[43]$} \\
\hline & & & & & 32.700 & 25.042 & 143.000 & 36.686 & [44] \\
\hline & & & & Zircaloy-4 & 681.864 & 32.3842 & - & - & {$[32]$} \\
\hline & & & & & 40.135 & 27.336 & 52.575 & 32.117 & {$[45]$} \\
\hline & & & & & 66.440 & 29.630 & 510.800 & 45.610 & {$[46]$} \\
\hline & & & & & 31.000 & 27.678 & 66.000 & 35.251 & {$[37]$} \\
\hline & & & & Zircaloy-2/4 & 98.7158 & 34.518 & - & - & {$[36]$} \\
\hline & & & & & 138.7460 & 34.469 & 106.4470 & 35.991 & {$[47]$} \\
\hline
\end{tabular}




\section{Liner Developments}

Liners are used on the interior surface of the cladding in BWRs to mitigate stress corrosion cracking and PCMI failures. The liners are typically made of slightly softer material than the Zircaloy-2 cladding, such as pure zirconium or a low tin content zirconium alloy (e.g., Zr-0.3\%Sn).

Prior to this project, the ability to model liners in BISON was limited to using Python mesh scripts in BISON to run the commercial software Cubit/Trelis [48] or using these commercial codes directly. While this possibility remains, it can be cumbersome for users. Therefore, work was completed to add support to the two internal mesh generators within BISON that are typically used within VERA to handle liners and improve ease-of-use. These mesh generators are SmearedPelletMeshGenerator and Layered1DMeshGenerator. In this chapter, we provide details on how to model liners using the two internal mesh generators as well as with the BISON Python mesh script. The mesh script should be reserved for more complex representations of the fuel rod (e.g., including dishes and chamfers in the fuel). A couple verification problems are also presented to verify that the creation of the internal meshes with liners was properly implemented.

\subsection{Internal Meshing Capabilities}

Internal meshing capabilities eliminate the need for third-party software to generate the geometries used in fuel performance analyses. By providing capabilities internal to BISON, a user can create the mesh directly in the input file used for the fuel performance simulation. For fuel rod analyses, two geometric representations are used, Layered1D, and 2D-RZ axisymmetric. In BISON, these geometries can be created by the Layered1DMeshGenerator and SmearedPelletMeshGenerator, respectively. These mesh generators existed in BISON prior to this work, just the capability to include liners in the meshes generated by them was added here.

\subsubsection{Layered1DMeshGenerator}

In a Layered1D representation of a fuel rod, discrete axial layers are used that each assume axisymmetry. Thermo-mechanics is solved in the radial direction in each layer with out-of-plane effects captured by the use of a generalized plane strain approach. Global fuel rod quantities as rod internal volume, fission gas released, and rod internal pressure are determined by summing the individual contributions from each layer. 
To support rapid sensitivity analysis the ability to model liners for 2D-RZ axisymmetric and Layered1D geometries has been added to the internal mesh generators in BISON.

Since the ability to model liners on the interior surface of the cladding was added, a broad range of meshes can be created using the Layered1DMeshGenerator. These include, fuel only, cladding only, fuel and cladding, fuel and cladding with a liner, fuel and cladding with a coating, fuel and cladding within a capsule, fuel and cladding with a liner and a coating, fuel and cladding with a liner within a capsule, fuel and cladding with a coating within a capsule, and fuel and cladding with a liner and a coating within a capsule. In all cases, the fuel can either be annular or solid or both. All fuel rod meshes can also contain either or both of upper and lower plenums. Fig. 3.1 presents an example of a Layered1D mesh containing fuel, liner, and cladding. Details of the block names, sidesets, and nodeset conventions created by the mesh generator can be found on the BISON documentation page for the Layered1DMeshGenerator.

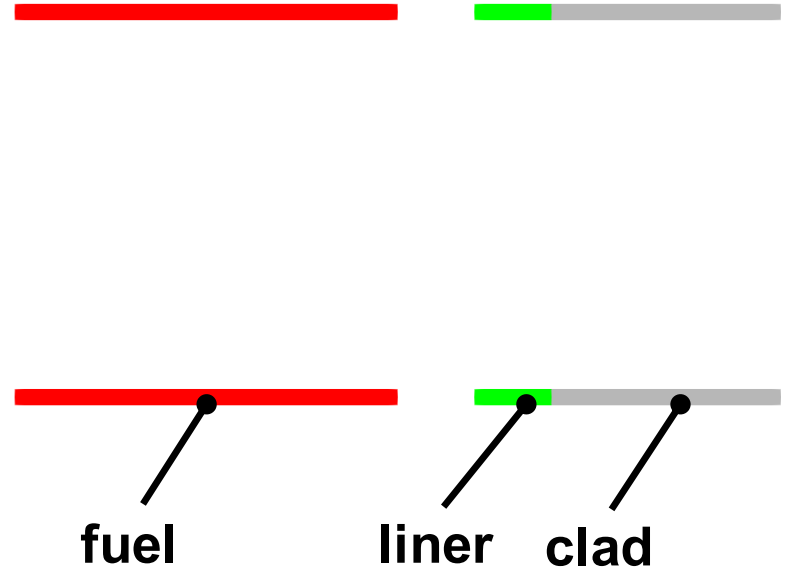

Figure 3.1. A Layered1D mesh containing fuel and lined cladding.

\subsubsection{SmearedPelletMeshGenerator}

In a smeared pellet representation of the fuel rod, a 2D-RZ axisymmetric geometry is assumed. The individual fuel pellets are not modeled, and the fuel stack is represented by a rectangle the height of the fuel stack. For many fuel performance calculations, this representation is sufficient. The same combinations of fuel, cladding, and liner as is available for Layered1D are possible, with the exception of including a capsule around the fuel rod. Fig. 3.2 presents an example of smeared pellet mesh containing fuel, liner, and cladding. Details 
of the block names, sidesets, and nodeset conventions created by the mesh generator can be found on the BISON documentation page for the SmearedPelletMeshGenerator [2].

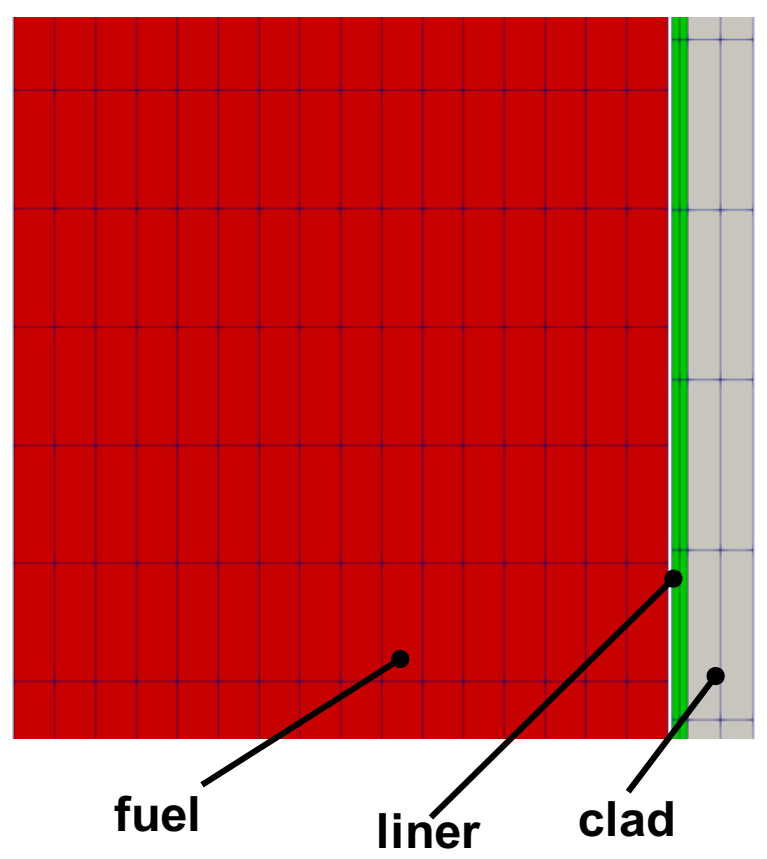

Figure 3.2. A smeared pellet mesh containing a lined cladding.

\subsection{BISON Mesh Script}

BISON supports an external Python mesh script that interfaces with the CUBIT software [48] that can include liners. The meshing script supports more complex meshes than that of the internal mesh generators such that distinct features of the fuel pellets (e.g., dishes and chamfers) can be captured. The mesh script can also be used to generate three-dimensional representations of the fuel rod. To use the script a user only has to modify the mesh_script_input.py file located at bison/tools/U02/ within the BISON repository. A portion of the mesh script is shown in the code snippet (see Listing 3.1). To include a liner in your mesh generated by the script the to parameter clad ['with_liner'] needs to be set to True and clad ['liner_width'] needs to be specified to a positive non-zero value, as seen in the code snippet. The other parameters are used to define the fuel characteristics and mesh densities. One can see that two different pellet types are defined, one that is solid with dishes and chamfers (Pellet1) and an annular pellet with flat end (Pellet2). The entire fuel stack is built using the pellets to insert the different pellet types in order from the bottom of the fuel stack to the top. In this example, one pellet with Pellet1 specifications is placed above and below a stack of ten pellets with Pellet2 specifications. The angle parameter defines the number of degrees swept in the azimuthal direction for 3D applications. A value of zero for angle results in 
a 2D mesh in the $\mathrm{x}-\mathrm{y}$ plane. Fig. 3.3 presents an example of a discrete mesh containing fuel with dishes and chamfers, a liner, and cladding.

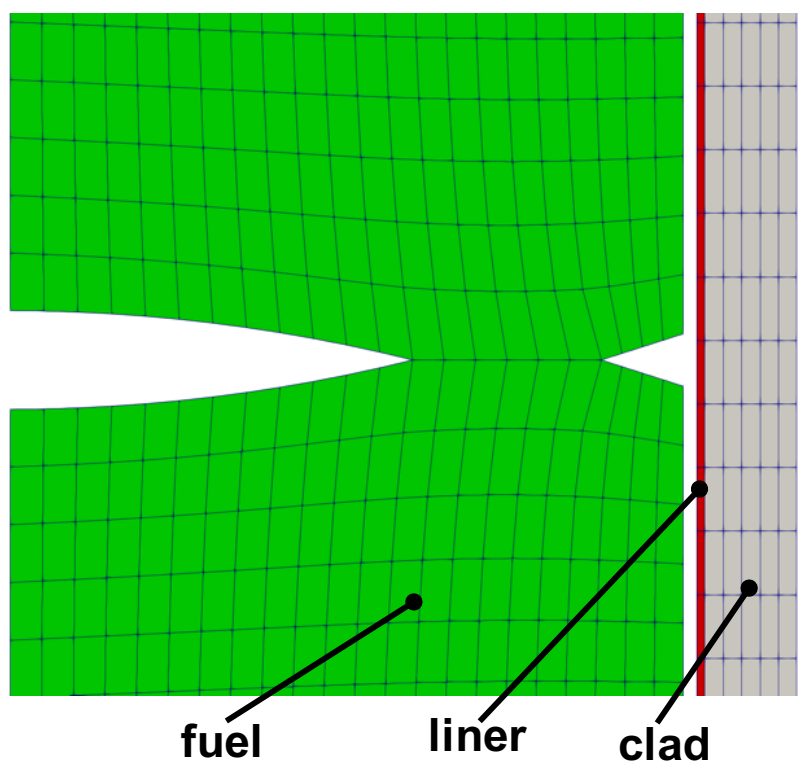

Figure 3.3. Discrete pellet mesh containing dishes and chamfers with a lined cladding.

\subsection{Verification}

If we consider constant isotropic solid properties, the temperature change across the gap, liner, and cladding can be obtained using the identity of continuous heat flux. This allows to obtain an analytic solution that is more representative of typical fuel rods. An infinitely long fuel rod has a constant thermal conductivity $k_{f}$ and internal heat generation $q^{\prime \prime \prime}$. It is exposed on its right surface to some constant temperature $T\left(r_{c o}\right)=T_{c o}$ [49]. The temperature jump across the gap, liner, and cladding is:

$$
T_{f}-T_{c o}=\frac{q^{\prime}}{2 \pi r_{f} h_{g a p}}+\frac{q^{\prime}}{2 \pi}\left\{\frac{1}{k_{l}} \ln \left(\frac{r_{c i}}{r_{l i}}\right)+\frac{1}{k_{c}} \ln \left(\frac{r_{c o}}{r_{c i}}\right)\right\}
$$

where $T_{f}$ is the fuel surface temperature, $T_{c o}$ is the cladding outside temperature, $k_{l}$ is the liner thermal conductivity, and $k_{c}$ is the cladding thermal conductivity.

The problem in this section focuses on testing the gap heat transfer for an open gap. To simplify the analytic solution, radiative heat transfer is ignored by setting emissivities to zero, and temperature jump distances are set to zero through BISON input. Thus, the gap heat transfer (see Appendix A) reduces to 
Listing 3.1. BISON mesh script example.

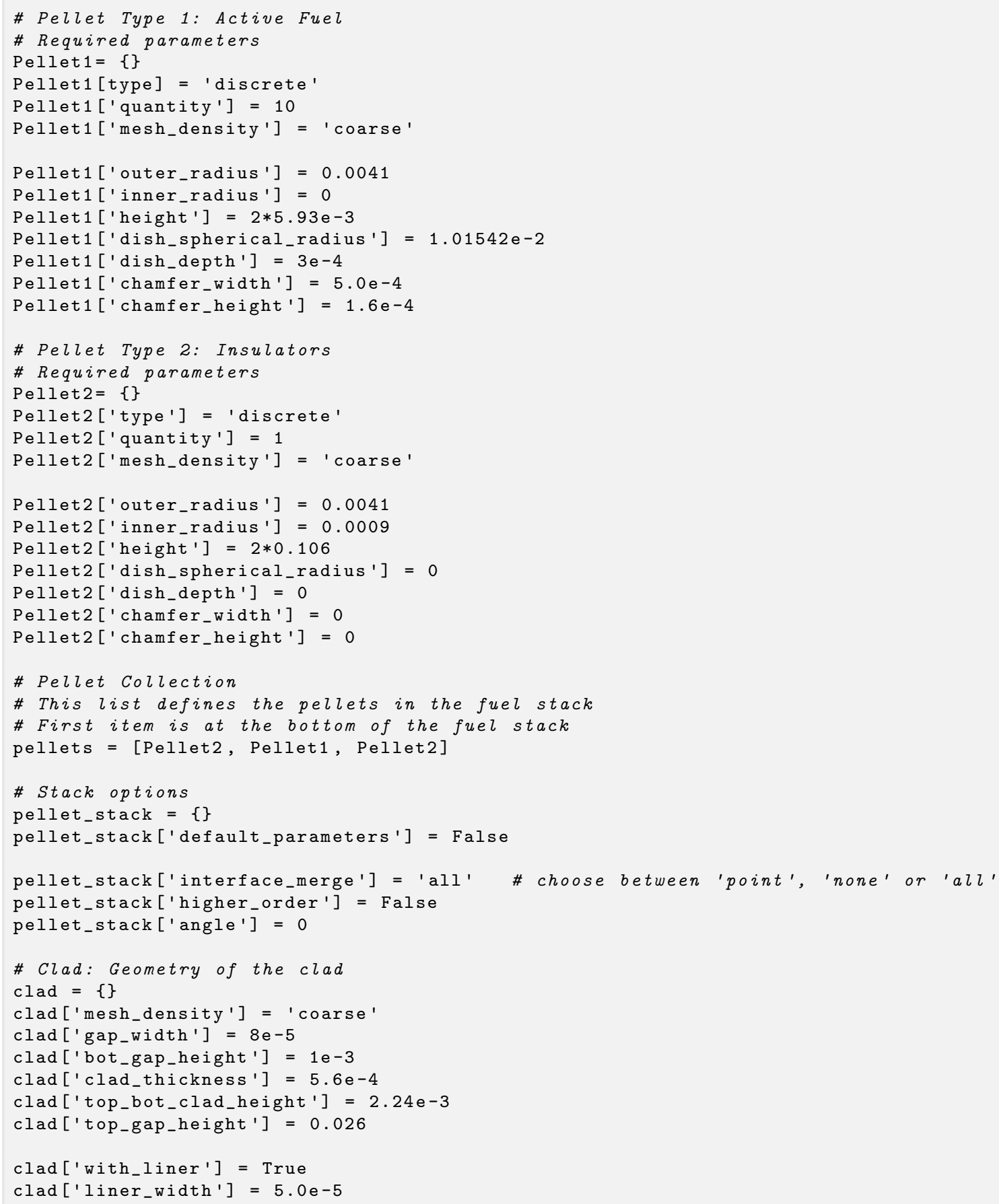


the fill gas conductance $h_{g}$ :

$$
h_{\text {gap }}=h_{g}=\frac{k_{g a s}}{r_{f} \ln \left(\frac{r_{l i}}{r_{f}}\right)}
$$

where $k_{\text {gas }}$ is the thermal conductivity of the fill gas and $r_{l i}$ is the liner inside radius. The analytic solution for the temperature distribution in the fuel is

$$
T(r)=T_{f}+\frac{q^{\prime}}{4 \pi k_{f}}\left(1-\frac{r^{2}}{r_{f}^{2}}\right),
$$

where $r_{f}$ is the fuel radius and $k_{f}$ is the fuel thermal conductivity.

The problem is run in BISON using three blocks: the fuel domain in $\vec{X} \in[0,1.0]$, the liner domain in $\vec{X} \in[1.0,1.05]$, and the clad domain in $\vec{X} \in[1.05,1.10]$. The domain between the fuel and liner blocks is filled with an inert gas with a predefined gas thermal conductivity. Neumann and Dirichlet boundary conditions are employed: $(d T / d r)_{r=0}=0$ and $T\left(r_{c o}\right)=T_{c o}$. The steady state heat conduction is considered with the following arbitrarily chosen constants: $k_{f}=k_{c}=10 \mathrm{~W} / \mathrm{m} / \mathrm{K}, k_{l}=1 \mathrm{~W} / \mathrm{m} / \mathrm{K}, k_{\text {gas }}=0.1 \mathrm{~W} / \mathrm{m} / \mathrm{K}$, and $q^{\prime \prime \prime}=400 \mathrm{~W} / \mathrm{m}^{3}$.

Fig. 3.4 shows the exact and computed finite element solutions for two FE types (linear or 1st-order: EDGE2; quadratic or 2nd-order: EDGE3). The exact and BISON results are in a good agreement, indicating that the new feature 'liner meshing' is correctly coded in BISON and functions as expected.

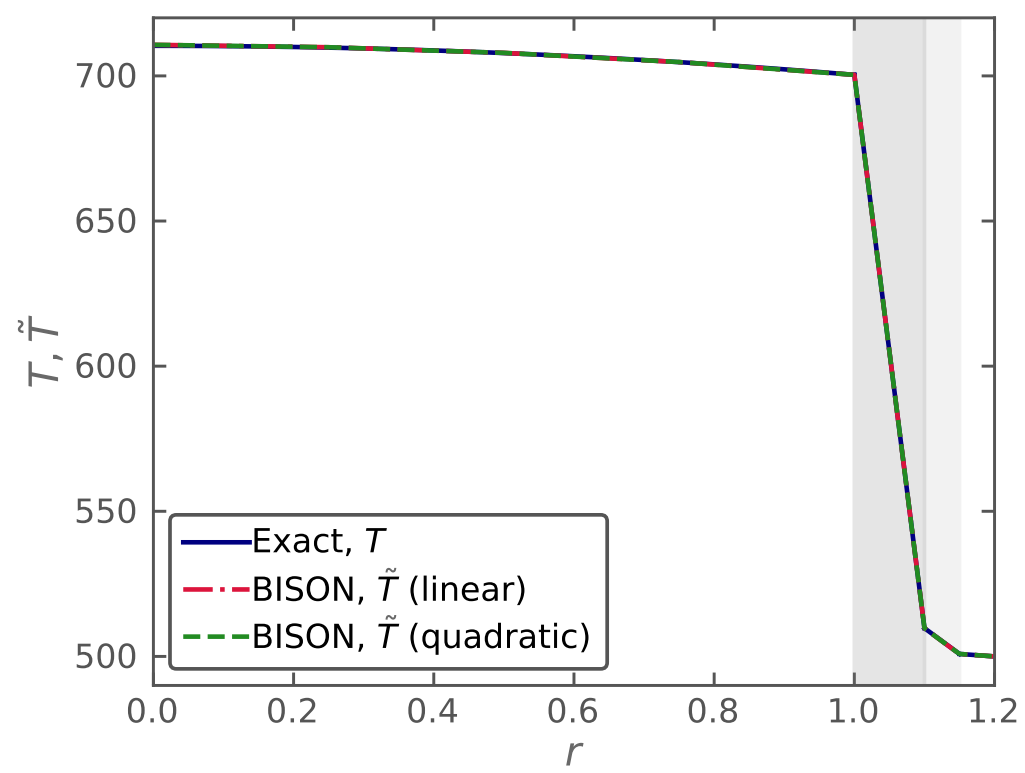

Figure 3.4. Temperature distribution for the verification problem. Exact and FE solutions are obtained using one-dimensional elements. 


\section{Validation}

This chapter focuses on the validation of new modeling options in BISON (from Chapter 2) by comparing code predictions to experimental measurements to determine the applicability of the model to real-world applications. Three rods selected were from the IFA-681.1 test series conducted at the Halden reactor in Norway. The tests investigated here include IFA-681.1 rods 1, 2, and 3. Details of the experimental measurements are described in Section 4.1. Then, BISON simulation settings for the aforementioned experimental data set are outlined in Section 4.2. Lastly, BISON's predictions are compared to the experimental data in Section 4.3, along with the discussion of results.

\subsection{Experiment: IFA-681 Rods 1, 2, and 3}

The Halden integral test IFA-681 was loaded in the Halden Boiling Water Reactor (HBWR) in January 2005 and operated till February 2012, with a completion of 15 cycles of irradiation. The rig contained two $\mathrm{UO}_{2}$ fuel rods and four $\mathrm{UO}_{2}-\mathrm{Gd}_{2} \mathrm{O}_{3}$ rods with varying gadolinia content [0.0, $8.0 \mathrm{wt} . \%$, which were manufactured by Institutt for Energiteknikk (IFE)-Kjeller [50]. An overview on the fuel rod specifictions of the IFA-681 is provided in Table 4.1. In practice, the rod designs were very similar, except for ${ }^{235} \mathrm{U}$ enrichment and $G d$ concentrations. The experiment was conducted under standard HBWR conditions, with the rig operating at a coolant pressure of approximately $3.5 \mathrm{MPa}$, and a coolant temperature of approximately $235^{\circ} \mathrm{C}$. Achieved burnup varied was around $31-45 \mathrm{MWd} / \mathrm{kgUO}_{2}$. The aim in the experiment was to evaluate the impact of $G d$ concentration to the oxide solution on the fuel thermal behavior, dimensional changes, and fission gas release.

The preliminary work was performed by [51] for the initial assessment of the BISON code for the modeling of $G d$-doped $\mathrm{UO}_{2}$ fuel. The analysis in BISON was performed for IFA-681.1 rods 1, 2, and 3 that were solid rods and were enriched to $4.70 \mathrm{wt} . \%^{235} U$ for rods 1 and 2 , and $2.80 \mathrm{wt} . \%^{235} U$ for rod 3 . The fuel and cladding specifications are tabulated in Table 4.1. Note that the top 11 pellets are hollow where the thermocouples were located. The integral responses from the experiment were obtained as follows: fuel temperature via a fuel centerline thermocouple, rod internal pressure via pressure transducer, fission gas release (FGR) via the online pressure measurements, and rod power via four neutron detectors install in the rig. 
Table 4.1. Fuel rod specifications for IFA-681 [52, 53, 54].

\begin{tabular}{l|c|c|c}
\hline Rod ID & Rod 1 & Rod 2 & Rod 3 \\
\hline Rod shape & solid & solid & solid \\
Fuel type & $\mathrm{UO}_{2}$ & $\mathrm{UO}_{2}-\mathrm{Gd}_{2} \mathrm{O}_{3}$ & $\mathrm{UO}_{2}-\mathrm{Gd}_{2} \mathrm{O}_{3}$ \\
$\mathrm{Gd}_{2} \mathrm{O}_{3}$ (wt.\%) & 0.0 & 2.0 & 8.0 \\
Enrichment (wt\% $\left.{ }^{235} \mathrm{U}\right)$ & 4.70 & 4.70 & 2.80 \\
Average grain size $(\mu \mathrm{m})$ & 10.9 & 7.3 & 8.4 \\
Active stack length $(\mathrm{m})$ & 400.4 & 400.0 & 400.2 \\
Pellet length (mm) & 10.25 & 9.92 & 9.97 \\
Pellet OD (mm) & 8.19 & 8.19 & 8.19 \\
Pellet ID (mm) & $0.0 / 1.8^{\star}$ & $0.0 / 1.8^{\star}$ & $0.0 / 1.8^{\star}$ \\
Pellet density (\%TD) & 95.6 & 96.1 & 96.0 \\
Cladding material & $\mathrm{Zy}-4$ & $\mathrm{Zy}-4$ & $\mathrm{Zy}-4$ \\
Cladding ID (mm) & 8.36 & 8.36 & 8.36 \\
Cladding OD (mm) & 9.50 & 9.50 & 9.50 \\
Diametral gap ( $\mu$ m) & 170 & 170 & 170 \\
Free volume (cm $\left.{ }^{3}\right)$ & 4.6 & 4.4 & 4.7 \\
Initial fill gas composition & helium & helium & helium \\
Fill gas pressure (MPa) & 1.0 & 1.0 & 1.0 \\
\hline Top 11 pellet only (thermocouple hole) & &
\end{tabular}

Top 11 pellet only (thermocouple hole)

\subsection{BISON Model Settings}

Two-dimensional, RZ-problem is setup for the BISON simulations of the IFA-681.1 Rods 1, 2, and 3. The supplementary histories, from Halden data, such linear heat rate (LHR), fast neutron flux, coolant pressure, and coolant inlet temperatures, are provided to BISON. Fuel Rod Analysis Toolbox is utilized for the condensation of the data prior to setting the BISON inputs. Radial power factors are computed by [51] at IFE-Halden Reactor Project via the HELIOS code $[55,56]$ to inform BISON analysis. Fig. 4.1 shows the rod average LHR for the aforementioned rods in this study.

\section{Geometry and Mesh}

The assumed geometry and mesh are shown in Figure 2 and Figure 3. The fuel pellet stack was modeled as a smeared column with merged insulator pellets (natural $\mathrm{UO}_{2}$ and $\mathrm{Al}_{2} \mathrm{O}_{3}$ ). The analysis in BISON was performed for the three out of six rods, IFA-681 rods 1, 2, and 3. Note that top 11 pellets are hollow where the thermocouple were located. The integral responses from the experiment were obtained as follows: fuel temperature via a fuel centerline thermocouple, rod internal pressure via pressure transducer, FGR via the online pressure measurements, and rod power via four neutron detectors installed in the rig. A two-dimensional axisymmetric geometry was used.

The rod consisted of $\mathrm{Al}_{2} \mathrm{O}_{3}$ hollow insulation pellets in the upper end of IFA- 681 rods 1, 2, and 3, followed by natural $\mathrm{UO}_{2}$ hollow pellets. The active fuel length consisted of 11 pellets of hollow enriched $\mathrm{UO}_{2}$ fuel, followed by $28 / 29$ pellets of the solid enriched $\mathrm{UO}_{2}$ fuel. Similarly, in the lower end of the rods, natural $\mathrm{UO}_{2}$ 
Figure 4.1. The average linear heat rate histories for the Halden IFA-681 irradiation.

solid pellets were followed by $\mathrm{Al}_{2} \mathrm{O}_{3}$ solid insulation pellets.

\section{Material and Behavioral Models}

$\mathbf{U O}_{2}-\mathbf{G d}_{2} \mathbf{O}_{3}$ fuel The following material and behavioral models were used for the fuel:

- ThermalFuel - Toptan [8]: For temperature and burnup dependent thermal properties.

- ComputeFiniteStrainElasticStress and ComputeIsotropicElasticityTensor: elastic mechanical behavior

- U02VolumetricSwellingEigenstrain: volumetric expansion due to solid and gaseous swelling

- U02RelocationEigenstrain: relocation strains

- ComputeThermalExpansionEigenstrain: thermal expansion with a constant instanteous thermal expansion coefficient

- Sifgrs: fission gas release model used with the gaseous swelling model U02VolumetricSwellingEigenstrain

Fuel-to-cladding gap The following behavioral models of GapConductanceLWR were turned on for the gap conductance modeling between the fuel and cladding:

- TOPTAN: gap conductance modeling according to $[57,58,59]$

- ADVANCED: gas thermal conductivity which takes gas pressure and temperature into account [60]

- TOPTAN: temperature jump distance model [57]

- TOPTAN: thermal accommodation model [57] 


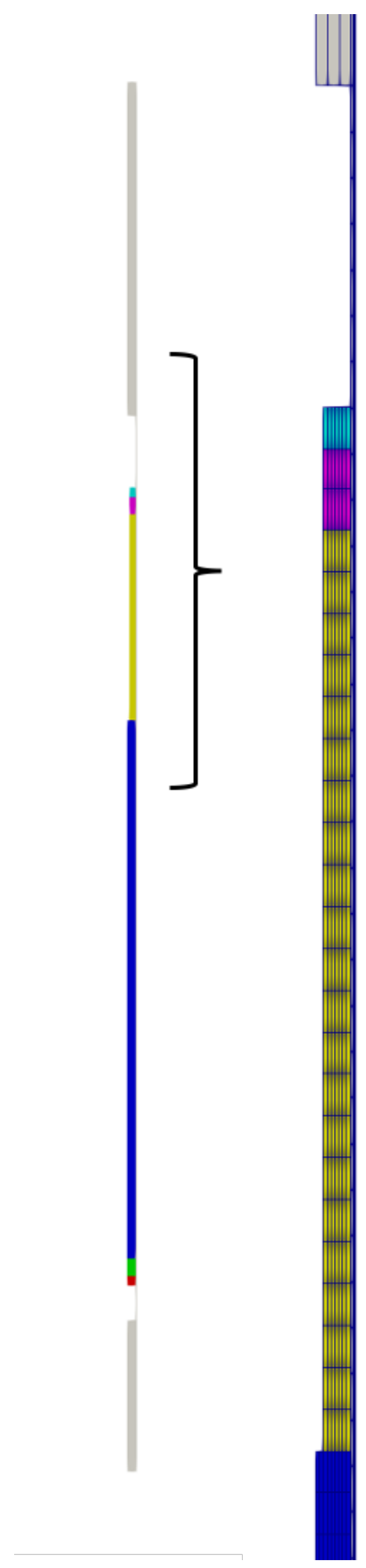

Figure 4.2. A front-view of the IFA-681.1 rod 1 (left) and a close-up view of the IFA-681.1 rod 1, a cut from the top of the fuel rod (right). 
Fuel-to-fuel gap The following behavioral models of GapConductance were utilized for the gap conductance modeling of the fuel-to-fuel gap at the thermocouple location considering a constant gas conductivity.

Zircaloy cladding For the clad material, a constant thermal conductivity of $16 \mathrm{~W} / \mathrm{m} / \mathrm{K}$ was used, and both thermal (primary and secondary) and irradiation creep were considered using the Limback creep model ([20]). The following material and thermal behavior models were used for the cladding:

- HeatConductionMaterial: thermophysical material properties

- ZryCreepLimbackHoppeUpdate and ZryElasticityTensor: mechanical creep and elastic deformation behavior

- ZryIrradiationGrowthEigenstrain: ESCORE model for volumetric swelling due to irradiation exposure

- ZryThermalExpansionMATPROEigenstrain: thermal expansion of Zircaloy with the MATPRO model

- Zry0xidation: corrosion oxide layer thickness

Natural $\mathbf{U O}_{2}$ material For the natural $\mathrm{UO}_{2}$ material, a constant thermal conductivity of $3.0 \mathrm{~W} / \mathrm{m} / \mathrm{K}$ and a constant specific heat capacity of $300 \mathrm{~J} / \mathrm{kg} / \mathrm{K}$ were used. The following material and thermal behavior models were used for the cladding:

- HeatConductionMaterial: Thermophysical material properties

- ComputeFiniteStrainElasticStress and ComputeIsotropicElasticityTensor: elastic mechanical behavior

- ComputeThermalExpansionEigenstrain: thermal expansion with a constant instantaneous thermal expansion coefficient

$\mathrm{Al}_{2} \mathrm{O}_{3}$ material For the $\mathrm{Al}_{2} \mathrm{O}_{3}$ material, a constant thermal conductivity of $18.0 \mathrm{~W} / \mathrm{m} / \mathrm{K}$ and a constant specific heat capacity of $880 \mathrm{~J} / \mathrm{kg} / \mathrm{K}$ were used. The following material and thermal behavior models were used for the cladding:

- HeatConductionMaterial: Thermophysical material properties

- ComputeFiniteStrainElasticStress and ComputeIsotropicElasticityTensor: elastic mechanical behavior

- ComputeThermalExpansionEigenstrain: thermal expansion with a constant instantaneous thermal expansion coefficient

Details and references for all of these models listed above can be found on the linked BISON documentation pages and in the provided inputs. 


\subsection{Results and Discussion}

BISON's predictions are compared to the experimental measurements for each rod in the following sections. A Python postprocessor script was used to extract the centerline temperature at the TC position. It is important to note that the thermocouples and pressure transducers failed, resulting in BISON going to the full time of the average linear heat rate (ALHR) while the experiment doesn't. BISON's temperature predictions are compared to the experimental data for IFA-681.1 Rods 1, 2, and 3 in Fig. 4.3. The new thermal conductivity model, along with the improved gap conductance modeling, results in a good agreement with the measured values for varied gadolinia-doped fuels.

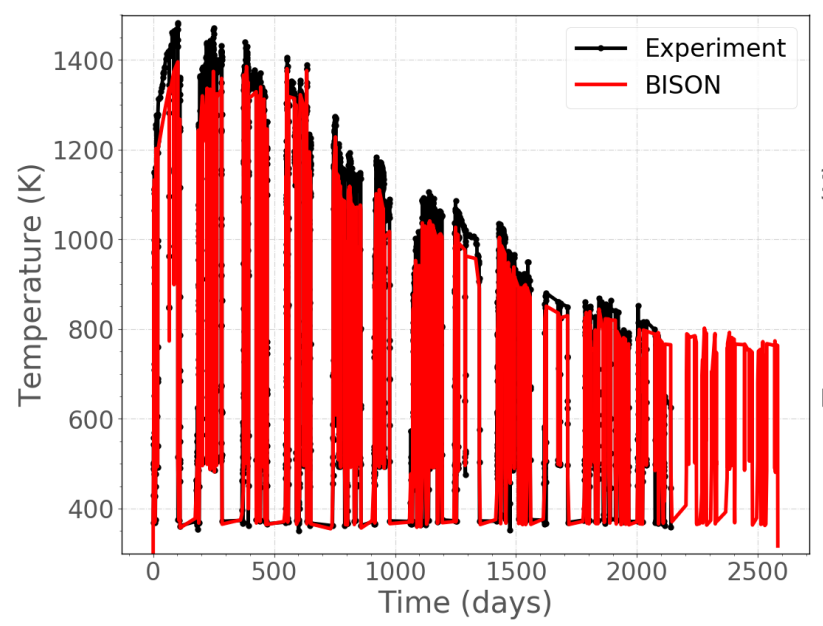

(a) IFA-681 Rod 1

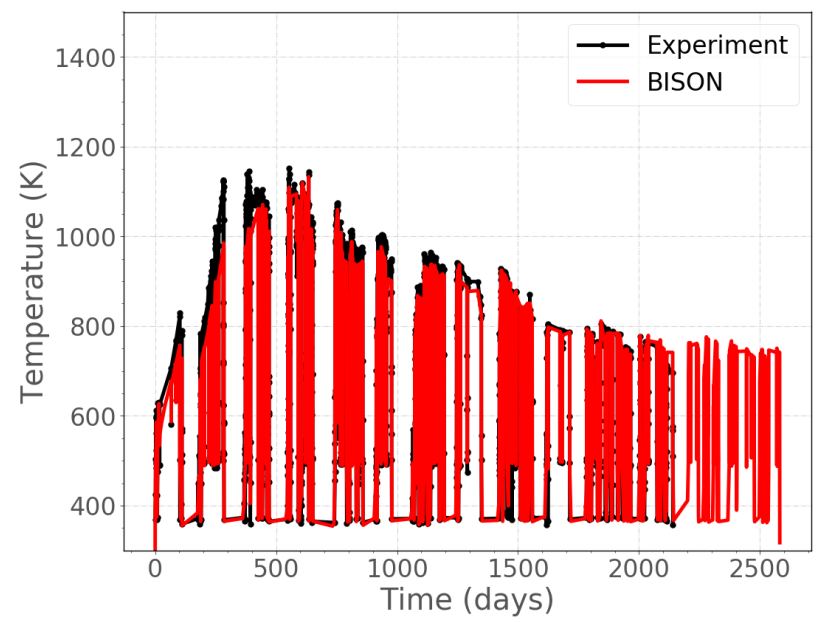

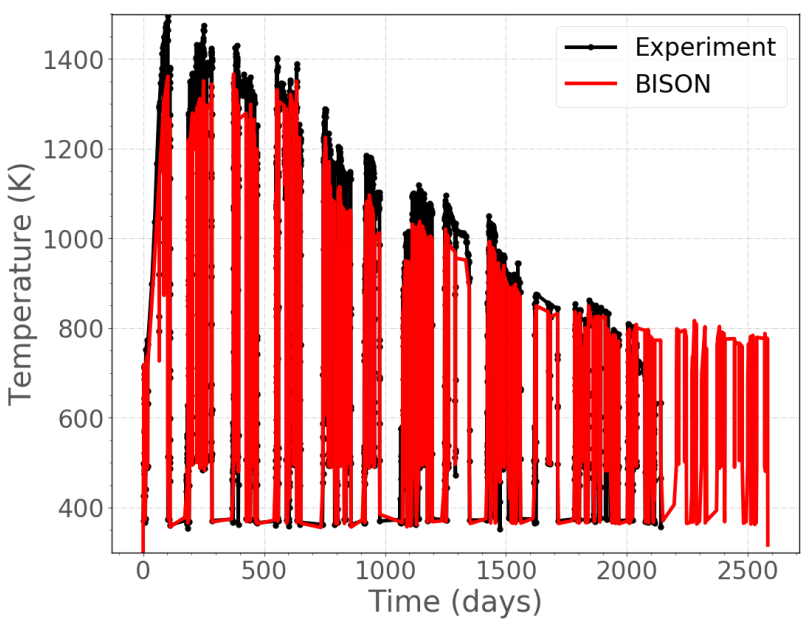

(b) IFA-681 Rod 2

(c) IFA-681 Rod 3

Figure 4.3. Halden IFA-681.1 fuel temperature histories at TC locations. 


\section{Concluding Remarks}

The BISON fuel performance code has been under development for approximately a decade. Over the years, developments have focused primarily on PWR materials, including potential ATF concepts as well as advanced reactor fuels such as metallic and TRISO fuel particles. The addition of the missing pieces for BWR analyses is required to support the funding opportunity announcement project entitled "Modeling \& Analysis of Exelon BWRs with VERA for Eigenvalue and Thermal Limits." The primary focus of this work was to develop the required material models that would aid in predicting cladding failure with a specific hydride distribution, considering the hydride geometry and its distribution within the liner cladding. The BWR modeling capabilities (see Chapter 2) that were made available or revisited in BISON are:

1. $\mathrm{UO}_{2}-\mathrm{Gd}_{2} \mathrm{O}_{3}$ properties (see Section 2.1): To account for the gadolinia addition to uranium dioxide fuel, two new thermal conductivity models - referred to as TOPTAN and STAICU for the typical burnup range of interest and the extended burnup applications, respectively - and one specific heat capacity model was implemented (made the default model for the specific heat capacity calculations). In addition to the implementation of new models, existing thermal conductivity models were reviewed again to confirm that the model implementations are correct, and regression tests are added for the models to protect their functionalities for the future code developments. These models are made available in ThermalFuel within BISON.

2. Pure zirconium properties (see Section 2.2): Pure zirconium is one of the materials used as a liner material in BWRs. The material property models for pure zirconium is scarce in the literature. The following models were found: thermal conductivity and specific heat capacity as thermal properties that are made available in ZrThermal of BISON and thermal expansion as the mechanical property, that is made available in ZrThermalExpansionEigenstrain within BISON.

3. Zirconium tin properties (see Section 2.3): Low tin content $(<1 \%)$ zirconium-tin alloys are another option used for BWR liners. As with pure zirconium, models for low tin content alloys for nuclear applications are scarce. The following model was found in the literature: thermal creep as a mechanical property that was made available in the ZrSnCreepUpdate model of BISON.

4. Zircaloy-2 properties (see Section 2.4): Zircaloy-2 is the cladding material of choice in BWRs. Without a specific model for liners, those of Zircaloy-2 are used. We documented the material models available already in BISON for Zircaloy-2 cladding: thermal conductivity and specific heat capacity as thermal properties (ThermalZry); and elastic constants (ZryElasticityTensor), thermal expansion 
(ZryThermalExpansionEigenstrain), thermal and irradiation creep (ZryCreepLOCAErbacherLimbackHoppeUpdate), and irradiation growth (ZryIrradiationGrowthEigenstrain) as mechanical properties.

5. Hydrogen migration/redistribution behavior model (see Section 2.5): BISON's existing model for the hydrogen migration and redistribution in the cladding was documented. Details of the up-to-date model (available) in the code were provided. Instructions were provided for the users on how to use this modeling option for a liner cladding geometry, along with some representative experimental values of the hydrogen diffusion and precipitation-dissolution kinetics of zirconium hydrides for pure zirconium, Zircaloy-2, and Zircaloy-4. A detailed analysis on the prediction of cladding failure with a specific hydride distribution is a primary focus of the next year's milestones.

Another task of this milestone was to develop liner meshing capabilities in BISON for BWR applications (see Chapter 3) to support ease-of-use and industry adoption of the code. Liners are used on the interior surface of the cladding in BWRs to mitigate stress corrosion cracking and PCMI failures. One can use external meshing tools such as Cubit/Trelis to create any type of geometry. To provide ease-of-use and to handle the mesh generation internally within BISON, the ability to handle liners was added to two exiting internal meshing capabilities:

1. Layered1DMeshGenerator models the fuel rod as an axial stack of one-dimensional radial analyses assuming azimuthal symmetry. Global quantities are computed by summing the individual contributions from the different layers. Meshes can include fuel, cladding, liner, and a capsule, or any combination of them.

2. SmearedPelletMeshGenerator models the fuel rod in a 2D-RZ axisymmetric representation with the fuel assumed to be a smeared column (i.e., right cylinder without dishes and chamfers). Meshes can include fuel, cladding, liner, and a capsule, or any combination of them.

3. Verification of the internal meshing capabilities was accomplished by simulating a simplistic thermal problem and demonstrating proper convergence behavior. This ensures that the internal meshing capabilities are generating properly connected meshes.

As a best practice of software quality assurance (SQA), all of these modeling features are documented, integrated into BISON, and run periodically to ensure that code capabilities are not lost as changes are made. This is achieved using regression tests that also serve as examples for the users how to use each modeling option in the code.

Validation is the comparison of model predictions to experimental measurements to determine the accuracy at which the simulations predict reality. Here, validation focused on the the Gd-bearing $\mathrm{UO}_{2}$ model developments, in particular, the new thermal conductivity models. Three rods from the IFA-681 series irradiated at the Halden reactory in Norway were analyzed. The rods were a non-doped $\mathrm{UO}_{2}$, a 2 wt.\% $\mathrm{Gd}_{2} \mathrm{O}_{3}$ and a 8 wt. $\% \mathrm{Gd}_{2} \mathrm{O}_{3}$ rod. Thermocouple temperature comparisons were in a very good agreement (see Chapter 4).

In addition to the aforementioned BWR material developments described in detail in this report, one journal article described BISON models for gadolinia-doped $\mathrm{UO}_{2}$, including verification and validation (see Chapter 6$)$. 
This work establishes a strong base for BISON's BWR modeling capabilities for Gd-doped $\mathrm{UO}_{2}$ fuels with lined cladding, which is to be expanded next year. In the future, this work will be expanded to include hydrogen and hydride embrittlement and its impact on failure predictions. Additional applications will be performed in BISON for BWR fuel behavior during normal operations (including control blade sequence exchanges involving down power and return to power maneuvers) and anticipated transients such as a reactivity insertion accident (RIA) driven by a control rod drop accident to examine whether PCMI is a potential failure mechanism and to identify what the failure limits should be for BWR cladding.

The milestones to be completed in FY2021 can be listed as:

1. to perform and document sensitivity analyses for BWR fuel (due 4/30/2021) and

2. to perform and document RIA analysis for BWR fuel (due 9/30/2021).

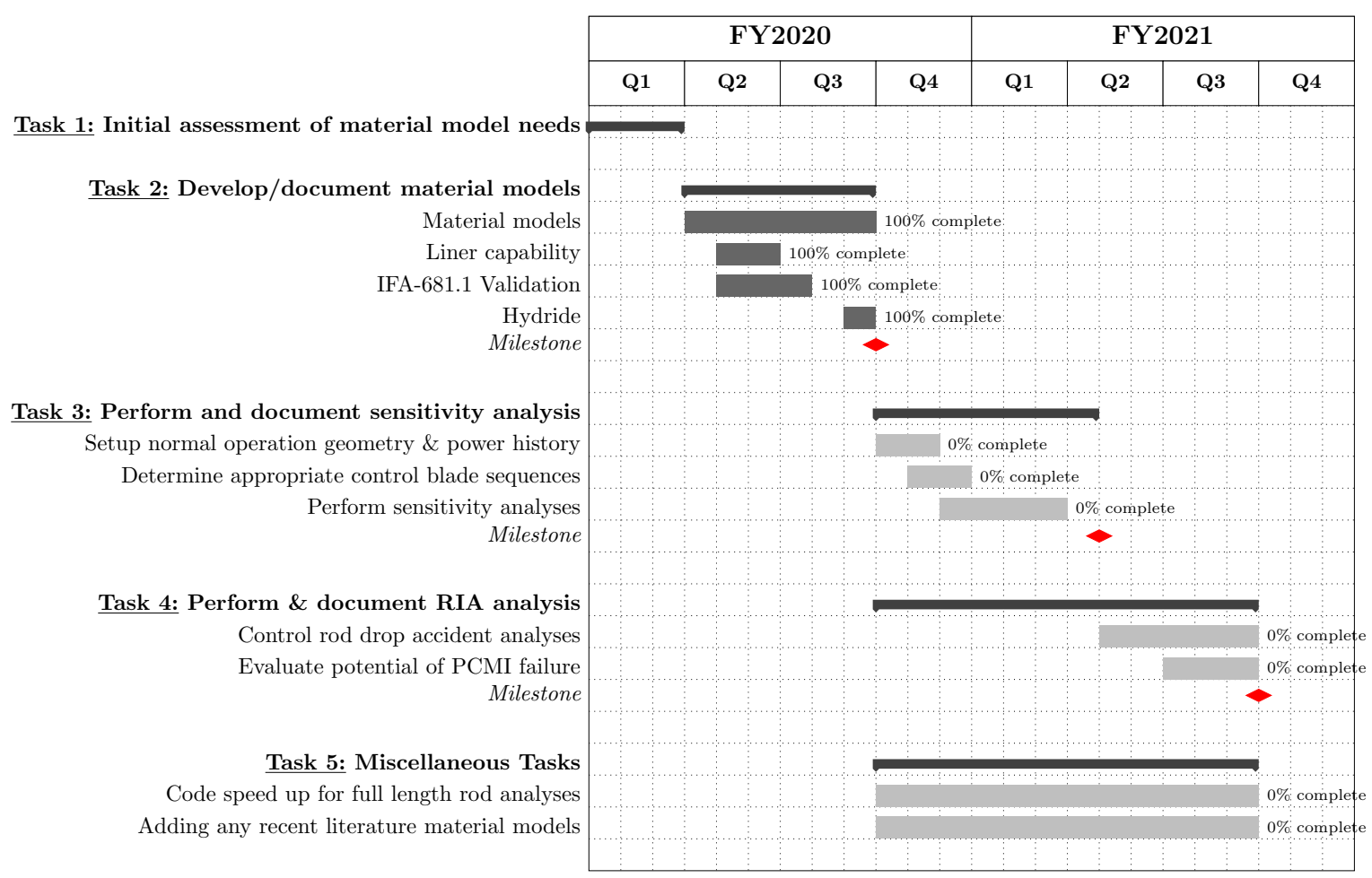

Figure 5.1. Gantt diagram for 2020-2021 fiscal years 


\section{Publications}

In regards to BWR capabilities in BISON, one journal article is under revision that illustrates the model development including validation. Highlights of the publication(s) are briefly provided as follows:

1. A. Toptan, G. Pastore, J.D. Hales, S. R. Novascone, R. L. Williamson, "Engineering-scale Modeling of Thermal Conductivity in BISON for $\mathrm{UO}_{2}$ and Gd-bearing $\mathrm{UO}_{2}$ Fuels," Journal of Nuclear Materials, 2020 (submitted, under review).

Highlights:

- An extensive literature survey for the development of an engineering-scale thermal conductivity model for uranium dioxide, including gadolinia-doping.

- Identified associated uncertainties in the models along with underlying assumptions.

- To assess the performance of thermal conductivity models, including the newly developed model in this study and the conventional thermal conductivity models commonly used in nuclear applications, the models are compared to each other (see Fig. 6.1) and to experimental data.

- A new postulated engineering-scale modeling strategy to allow flexibility in updating the thermal conductivity component for extended burnups. 
Figure 6.1. Comparisons of model predictive envelopes for the stoichiometric $\mathrm{UO}_{2}$ thermal conductivity in (a) the burnup range varied from 0 to $60 \mathrm{MWd} / \mathrm{kgU}$ and (b) the gadolinia concentration varied from 0 to $10 \mathrm{wt} \%$. The predictions from the proposed model (see TOPTAN model in Property 2.1; referred to as Eq.(6) on each plot) are plotted in each plot for ease of comparison with the other thermal conductivity models. Note that addition of either burnup or gadolinia degrades the thermal conductivity; therefore, the upper bound represents the non-doped fuel at zero burnup, and the lower bound represents the irradiated and Gd-doped fuel. 


\section{Bibliography}

[1] R. L. Williamson et al. "Multidimensional multiphysics simulation of nuclear fuel behavior". In: Journal of Nuclear Materials 423.1-3 (2012), pp. 149-163. DOI: 10.1016/j.jnucmat.2012.01.012.

[2] J. D. Hales et al. BISON Theory Manual The Equations Behind Nuclear Fuel Analysis. Idaho National Laboratory. Idaho Falls, Idaho, 2014.

[3] R. L. Williamson, J. D. Hales, et al. "BISON: A flexible code for advanced simulation of the performance of multiple nuclear fuel forms". In: Nuclear Technology (2020). submitted.

[4] D. Gaston et al. "MOOSE: A parallel computational framework for coupled systems of nonlinear equations". In: Nuclear Engineering and Design 239.10 (2009), pp. 1768-1778. DOI: 10 . 1016 / j . nucengdes.2009.05.021.

[5] C.J. Permann et al. "MOOSE: Enabling massively parallel multiphysics simulation". In: SoftwareX 11 (2020), p. 100430. DOI: 10.1016/j.softx.2020.100430.

[6] J. D. Hales et al. "Multidimensional multiphysics simulation of TRISO particle fuel". In: Journal of Nuclear Materials 443 (2013), pp. 531-543. DOI: 10.1016/j . jnucmat.2013.07.070.

[7] J. D. Hales et al. BISON TRISO Modeling Advancements and Validation to AGR-1 Data. PEMP Notable Outcome 1.1.C Completion Report INL/EXT-20-00297. Idaho National Laboratory, July 2020.

[8] A. Toptan et al. "Engineering-scale modeling of thermal conductivity in BISON for $\mathrm{UO}_{2}$ and Gd bearing $\mathrm{UO}_{2}$ fuels". In: Journal of Nuclear Materials (2020). (submitted).

[9] J. C. Maxwell. Treatise on Electricity and Magnetism. Oxford University Press Oxford, 1873/1904.

[10] G. Ondracek and B. Schultz. "The porosity dependence of the thermal conductivity for nuclear fuels". In: Journal of Nuclear Materials 46.3 (1973), pp. 253-258. DOI: 10.1016/0022-3115(73)90039-1.

[11] C. Ronchi et al. "Effect of burn-up on the thermal conductivity of uranium dioxide up to 100.000 MWd $\mathrm{t}^{-1}$ ". In: Journal of Nuclear Materials 327.1 (Apr. 2004), pp. 58-76. DOI: 10.1016/j.jnucmat. 2004. 01.018.

[12] D. Staicu et al. "Effect of burn-up on the thermal conductivity of uranium-gadolinium dioxide up to 100 GWd/tHM". In: Journal of Nuclear Materials 453 (2014), pp. 259-268. DOI: 10.1016/j .jnucmat. 2014.07 .006 . 
[13] D. Staicu. "2.17 - Thermal Properties of Irradiated $\mathrm{UO}_{2}$ and MOX". In: Comprehensive Nuclear Materials 2 (2012), pp. 439-464. DOI: 10.1016/B978-0-08-056033-5.00038-0.

[14] D.D. Lanning, C.E. Beyer, and K.J. Geelhood. FRAPCON-3 Updates, including Mixed-Oxide fuel properties. Tech. rep. NUREG/CR-6534, Vol.4; PNNL-11513. Richland, WA 99532: Pacific Northwest National Laboratory, 2005. URL: https://www.nrc.gov/docs/ML0514/ML051440720.pdf.

[15] A. Marion. Letter dated June 13, 2016 to HN Berkow (USNRC/NRR). Tech. rep. Nuclear Energy Institute (NEI), 2006. URL: https://www.nrc.gov/docs/ML0616/ML061650107.pdf.

[16] W. F. Lyon. Summary report: Gd thermal conductivity model updates. Tech. rep. ANA-P1400138-TN03 Rev. 2. Anatech Corp., 2015.

[17] W.J. Luscher, K.J. Geelhood, and I.E. Porter. Material Property Correlations: Comparisons between FRAPCON-4.0, FRAPTRAN-2.0, and MATPRO. Tech. rep. PNNL-19417 Rev. 2. Pacific Northwest National Laboratory, Sept. 2015.

[18] J.K. Fink and L. Leibowitz. "Thermal conductivity of zirconium". In: Journal of Nuclear Materials 226.1-2 (1995), pp. 44-50. DOI: 10.1016/0022-3115(95)00110-7.

[19] S.K. Jha, S. Dixit, and D. Srivastava. "Study of Thermomechanical Behavior of Zirconium-0.3 Tin Alloy". In: Journal of Materials Engineering and Performance 28 (2019), pp. 5127-5137. DOI: 10 . $1007 /$ s11665-019-04204-x.

[20] M. Limbäck and T. Andersson. "A Model for Analysis of the Effect of Final Annealing on the In- and Out-of-Reactor Creep Behavior of Zircaloy Cladding". In: Zirconium in the Nuclear Industry: Eleventh International Symposium. ASTM STP 1295. 1996, pp. 448-468.

[21] F. J. Erbacher et al. "Burst criterion of Zircaloy fuel claddings in a loss-of-coolant accident". In: Zirconium in the Nuclear Industry, Fifth Conference, ASTM STP 754, D.G. Franklin Ed. American Society for Testing and Materials. 1982, pp. 271-283.

[22] Q. Auzoux et al. "Hydride reorientation and its impact on ambient temperature mechanical properties of high burn-up irradiated and unirradiated recrystallized Zircaloy-2 nuclear fuel cladding with an inner liner". In: Journal of Nuclear Materials 494 (2017), pp. 114-126. DOI: 10.1016/j . jnucmat. 2017.07 .022 .

[23] E. Nagase and T. Fuketa. "Influence of Hydride Re-orientation on BWR Cladding Rupture under Accidental Conditions". In: Journal of Nuclear Science and Technology 41.12 (2004), pp. 1211-1217. DOI: $10.1080 / 18811248.2004 .9726350$.

[24] D. S. Stafford. "Multidimensional simulations of hydrides during fuel rod lifecycle". In: Journal of Nuclear Materials 466 (2015), pp. 362-372. DOI: 10.1016/j.jnucmat.2015.06.037.

[25] Z. Aly et al. "Variance-based sensitivity analysis applied to hydrogen migration and redistribution model in Bison. Part I: Simulation of historical experiments". In: Journal of Nuclear Materials 524 (2019), pp. 90-100. DOI: 10.1016/j.jnucmat.2019.06.035. 
[26] Z. Aly et al. "Variance-based sensitivity analysis applied to the hydrogen migration and redistribution model in Bison. Part II: Uncertainty quantification and optimization". In: Journal of Nuclear Materials 523 (2019), pp. 478-489. DOI: 10.1016/j.jnucmat.2019.06.023.

[27] Florian Passelaigue. Hydride Nucleation - Growth - Dissolution Model: Implementation in BISON. 2020. URL: https://etda.libraries.psu.edu/catalog/17572fpp8.

[28] G. Pastore et al. Hydrogen Migration and Redistribution in BISON. Tech. rep. INL/EXT-20-00214. Idaho National Lab.(INL), Apr. 2020.

[29] J. Kearns. "Diffusion coefficient of hydrogen in alpha zirconium, Zircaloy-2 and Zircaloy-4". In: Journal of Nuclear Materials 43.3 (1972), pp. 330-338. DOI: 10.1016/0022-3115(72)90065-7.

[30] C.M. Schwartz and M.W. Mallett. In: Trans. Amer. Soc. Metals. Vol. 46. 1954, pp. 640-654.

[31] M. Someno and N.K. Gakkaishi. "Solubility and diffusion of hydrogen in zirconium". In: J. Japan Inst. Metals 24.4 (1960). (in Japanese), pp. 249-253. URL: https://www.jim.or.jp/journal/j/24/04/ 249-253.html.

[32] E.A. Gulbransen and K.F. Andrew. "Solubility and Decomposition Pressures of Hydrogen in AlphaZirconium". In: Trans. AIME 203. Vol. 7. 1955, pp. 136-144. DOI: 10.1007/BF03377469.

[33] M.W. Mallett and W.M. Albrecht. "Low-Pressure Solubility and Diffusion of Hydrogen in Zirconium". In: Journal of The Electrochemical Society 104.3 (1957). URL: https : / iopscience . iop . org / article/10.1149/1.2428522.

[34] A. Sawatzky. "The diffusion and solubility of hydrogen in the alpha phase of zircaloy-2". In: Journal of Nuclear Materials 2.1 (1960), pp. 62-68. DOI: 10.1016/0022-3115(60)90025-8.

[35] T.B. Douglas. "The Zirconium-Hydrogen System: Some Thermodynamic Properties from a Heat Content Study". In: Journal of the American Chemical Society 80.19 (1958), pp. 5040-5046. DOI: 10 . 1021/ja01552a010.

[36] J.J. Kearns. "Terminal solubility and partitioning of hydrogen in the alpha phase of zirconium, Zircaloy2 and Zircaloy-4". In: Journal of Nuclear Materials 22.3 (1967), pp. 292-303. DOI: 10.1016/0022$3115(67) 90047-5$.

[37] B. Kammenzind et al. "Hydrogen pickup and redistribution in alpha-annealed Zircaloy-4". In: E.R. Bradley, G.P. Sabol (Eds.), Zirconium in the Nuclear Industry: Eleventh International Symposium, ASTM STP 1295. 1996, pp. 338-370.

[38] G.Östberg. "Determination of hydride solubility in alpha phase zirconium, zircaloy-2 and zircaloy-4". In: Journal of Nuclear Materials 5.2 (1962), pp. 208-215. DOI: 10.1016/0022-3115(62)90101-0.

[39] W.H. Erickson and D. Hardie. "The influence of alloying elements on the thermal solubility of hydrogen in $\alpha$-zirconium". In: Journal of Nuclear Materials 13.2 (1964), pp. 254-262.

[40] A. Sawatzky. "The heat of transport of hydrogen in zirconium alloys". In: Journal of Nuclear Materials 9.3 (1963), p. 364. DOI: 10.1016/0022-3115(63) 90154-5. 
[41] K. Une and S. Ishimoto. "Terminal Solid Solubility of Hydrogen in Unalloyed Zirconium by Differential Scanning Calorimetry". In: Journal of Nuclear Science and Technology 41.9 (2004), pp. 949-952. DOI: 10.1080/18811248.2004.9715569.

[42] K. Une and S. Ishimoto. "Dissolution and precipitation behavior of hydrides in Zircaloy-2 and high Fe Zircaloy". In: Journal of Nuclear Materials 322.1 (2003), pp. 66-72. DOI: 10.1016/S0022-3115(03) 00320-9.

[43] K.B. Colas. Fundamental Experiments on Hydride Reorientation in Zircaloy. 2012.

[44] K. Une et al. "The terminal solid solubility of hydrogen in irradiated Zircaloy-2 and microscopic modeling of hydride behavior". In: Journal of Nuclear Materials 389.1 (2009), pp. 127-136. DOI: 10 . 1016/j.jnucmat.2009.01.017.

[45] R. Tang and X. Yang. "Dissolution and precipitation behaviors of hydrides in N18, Zry-4 and M5 alloys". In: Int. J. Hydrogen Energy 34.17 (2009), pp. 7269-7274. DOI: 10.1016/j . i jhydene. 2009 . 07.018 .

[46] O. Zanellato, M. Preuss, J.-Y. Buffiere, et al. "Synchrotron diffraction study of dissolution and precipitation kinetics of hydrides in zircaloy-4". In: Journal of Nuclear Materials 420.1-3 (2012), pp. 537-547. DOI: $10.1016 / j \cdot$ jnucmat.2011.11.009.

[47] A. McMinn, E. Darby, and J. Schofield. "The terminal solid solubility of hydrogen in zirconium alloys". In: Zirconium in the Nuclear Industry: 12th International Symposium. 2000, pp. 173-195.

[48] M. Skroch et al. CUBIT: Geometry and Mesh Generation Toolkit, 15.5 User Documentation. Tech. rep. SAND2019-3478W. Sandia National Laboratories, 2019.

[49] A. Toptan et al. FY20 Verification of BISON Using Analytic and Manufactured Solutions. Tech. rep. CASL-U-2020-1939-000; SAND2020-3887R. Sandia National Lab.(SNL), 2020. DOI: 10.2172/1614683.

[50] J. D. Hales et al. "Solving Nonlinear Solid Mechanics Problems with the Jacobian-Free Newton Krylov Method". In: Computer Modeling in Engineering \& Sciences 84.2 (2012), pp. 123-154. DOI: 10.3970/ cmes. 2012.084.123.

[51] G. Pastore et al. "Modelling of the Galolinium Fuel Test IFA-681 using the BISON code". In:

[52] F. Khattout. The Gadolinia Fuel Test IFA-681: Overview of In-Pile Measurements From Beginning of Irradiation to Unloading. Tech. rep. HWR-1038. OECD Halden Reactor Project, 2013.

[53] V. Colombier et al. Comparative Study of the Gadolinia Experiments IFA-515.10, IFA-636, IFA-681.1, and IFA-676.1. Tech. rep. HWR-924. OECD Halden Reactor Project, 2010.

[54] Institutt for Energiteknikk OECD Halden Reactor Project. Data Sheet / IFA-681.1, Technical Note. 2005.

[55] R. J. J. Stammler (Ed.) HELIOS documentation. Tech. rep. Norway: FMS Fuel Management System, Studsvik Scandpower, 2000. 
[56] J.-C. Kim. Physics calculations for IFA-681.1. Tech. rep. CP note 03-51. OECD Halden Reactor Project, 2003.

[57] A. Toptan, D. J. Kropaczek, and M. N. Avramova. "Gap conductance modeling I: Theoretical considerations for single- and multi-component gases in curvilinear coordinates". In: Nuclear Eng Design 353 (2019). DOI: $10.1016 / j$.nucengdes . 2019.110283.

[58] A. Toptan, D. J. Kropaczek, and M. N. Avramova. "Gap conductance modeling II: Optimized model for $\mathrm{UO}_{2}$-Zircaloy interfaces". In: Nuclear Eng Design 355 (2019). DOI: 10.1016/j . nucengdes . 2019 . 110289.

[59] A. Toptan et al. "Modeling of gap conductance for LWR fuel rods applied in the BISON code". In: Journal of Nuclear Science and Technology 57.8 (2020), pp. 963-974. DOI: 10.1080/00223131.2020. 1740808 .

[60] A. Toptan, D. J. Kropaczek, and M. N. Avramova. "On the Validity of the Dilute Gas Assumption for Gap Conductance Calculations in Nuclear Fuel Performance Codes". In: Nuclear Eng Design 350 (2019), pp. 1-8. DOI: 10.1016/j .nucengdes.2019.04.042.

[61] A. Toptan. "A Novel Approach to Improve Transient Fuel Performance Modeling in Multi-Physics Calculations". PhD thesis. North Carolina State University, 2019. URL: https://repository. lib. ncsu.edu/handle/1840.20/36352. 


\section{A. Gap Conductance: Thermal Aspects}

The temperature jump across the gap in BISON is computed as a summation of heat transfers: fill gas conductance $h_{g}$, contact conductance $h_{c}$, and radiative conductance $h_{r}[2,60,57,58,59,61]$ :

$$
h_{\text {gap }}=h_{g}+h_{c}+h_{r}
$$

where

$$
\begin{aligned}
& h_{g}=\left\{\begin{array}{cc}
\frac{k_{\text {gas }}}{d+g_{1}+g_{2}} & \text { plane } \\
\frac{k_{\text {gas }}}{r_{1}\left(\ln \left(\frac{r_{2}}{r_{1}}\right)+\frac{g_{1}}{r_{1}}+\frac{g_{2}}{r_{2}}\right)} & \text { cylinder } \\
\frac{k_{\text {gas }}}{r_{1}^{2}\left(\left[\frac{1}{r_{1}}-\frac{1}{r_{2}}\right]+\frac{g_{1}}{r_{1}^{2}}+\frac{g_{2}}{r_{2}^{2}}\right)} & \text { sphere }
\end{array},\right. \\
& h_{c}=C \frac{\Lambda}{r_{a}}\left(\frac{W}{H}\right) \text {, } \\
& h_{r}=\frac{\sigma_{S B}\left(T_{1}^{2}+T_{2}^{2}\right)\left(T_{1}+T_{2}\right)}{\frac{1}{\varepsilon_{1}}+\left(\frac{1}{\varepsilon_{2}}-1\right)} .
\end{aligned}
$$

where $k_{\text {gas }}$ is the fill gas thermal conductivity, $d$ is the gap distance, $g_{i}$ is the temperature jump distance, $\Lambda$ is the harmonic mean of thermal conductivities of the surrounding solids, $r_{a}$ is the contact radius that is defined as a function of the surface roughness, $W$ is the load on the contact interface, $H$ is the Meyer's hardness of the softer material, $\sigma_{S B}$ is the Stefan-Boltzmann constant, $T_{i}$ is temperature, $\varepsilon_{i}$ is the emissivity, and $r_{i}$ is the radius for the solid bodies $i=1,2\left(r_{1}<r_{2}\right)$. 